\title{
Radio counterpart of the lensed submm emission in the cluster MS0451.6-0305: new evidence for the merger scenario
}

\author{
A. Berciano Alba ${ }^{1,2}$, L. V. E. Koopmans ${ }^{2}$, M. A. Garrett ${ }^{1,3,4}$, O. Wucknitz ${ }^{5}$, and M. Limousin ${ }^{6,7}$ \\ 1 Netherlands Foundation for Research in Astronomy (ASTRON), Postbus 2, 7990 AA Dwingeloo, The Netherlands \\ e-mail: berciano@astro.rug.nl \\ 2 Kapteyn Astronomical Institute, University of Groningen, PO Box 800, 9700 AV, Groningen, The Netherlands \\ 3 Leiden Observatory, PO Box 9513, 2300 RA, Leiden, The Netherlands \\ ${ }^{4}$ Centre for Astrophysics and Supercomputing, Swinburne University of Technology, Mail number H39, PO Box 218, \\ Hawthorn, Victoria 3122, Australia \\ 5 Argelander-Institut für Astronomie (AIfA), University of Bonn, Auf dem Hügel 71, 53121 Bonn, Germany \\ ${ }^{6}$ Laboratoire d'Astrophysique de Marseille, UMR 6110, CNRS-Université de Provence, 38 rue Frédéric Joliot-Curie, \\ 13388 Marseille Cedex 13, France \\ 7 Dark Cosmology Centre, Niels Bohr Institute, University of Copenhagen, Juliane Maries Vej 30, 2100 Copenhagen, Denmark
}

Received 16 July 2009 / Accepted 7 September 2009

\section{ABSTRACT}

\begin{abstract}
Context. SMM J04542-0301 is an extended $\left(\sim 1^{\prime}\right)$ submm source located near the core of the cluster MS0451.6-0305. It has been suggested that part of its emission arises from the interaction between a LBG and two EROs at $z \sim 2.9$ that are multiply-imaged in the optical/NIR observations. However, the dramatic resolution difference between the sub-mm map and the optical/NIR images make it difficult to confirm this hypothesis.

Aims. In a previous paper, we reported the detection of $1.4 \mathrm{GHz}$ continuum radio emission coincident with this sub-mm source using VLA archival data. To fully understand the relation between this radio emission, the sub-mm emission, and the optical/IR multiplyimaged sources, we have re-observed the cluster with the VLA at higher resolution.

Methods. The previous archival data has been re-reduced and combined with the new observations to produced a deep $\left(\sim 10 \mu \mathrm{Jy}_{\text {beam }}^{-1}\right)$, high resolution $\left(\sim 2^{\prime \prime}\right)$ map centred on the cluster core. The strong lensing effect in the radio data has been quantified by constructing a new lens model of the cluster.

Results. From the high resolution map we have robustly identified six radio sources located within SMM J 04542-0301. The brightest and most extended of these sources (RJ) is located in the middle of the sub-mm emission, and has no obvious counterpart in the optical/NIR. Three other detections (E1, E2 and E3) seem to be associated with the images of one of the EROs (B), although the NIR and radio emission appear to originate at slightly different positions in the source plane. The last two detections (CR1 and CR2), for which no optical/NIR counterpart have been found, seem to constitute two relatively compact emitting regions embedded in a $\sim 5^{\prime \prime}$ extended radio source located at the position of the sub-mm peak. The presence of this extended component (which contributes $38 \%$ of the total radio flux in this region) can only be explained if it is being produced by a lensed region of dust obscured star formation in the center of the merger. A comparison between the radio and sub-mm data at the same resolution suggests that E1, E2, E3, CR1 and CR2 are associated with the sub-mm emission.

Conclusions. The radio observations presented in this paper provide strong observational evidence in favour of the merger hypothesis. However, the question if RJ is also contributing to the observed sub-mm emission remains open. These results illustrate the promising prospects for radio interferometry and strong gravitational lensing to study the internal structure of SMGs.
\end{abstract}

Key words. galaxies: clusters: individual: SMM J 04542-0301 - galaxies: starburst - radio continuum: galaxies galaxies: interactions - gravitational lensing: strong

\section{Introduction}

The detection of the cosmic infrared background (CIB) by the COBE satellite (Puget et al. 1996; Hauser et al. 1998) established that about half of the total radiation in the universe comes from dust-obscured galaxies that are missing from optical surveys (see Lagache et al. 2005, for a review). This population of dusty objects was first resolved by the IRAS and ISO satellites up to $z \sim 1$, and turned out to be dominated by luminous and ultra-luminous IR galaxies (LIRGs and ULIRGs). The step into the high- $z$ universe came with the advent of sub-mm and $\mathrm{mm}$ surveys, since the far-infrared (FIR) luminosity peak of high- $z$ obscured galaxies is red-shifted into the sub-mm band (Franceschini et al. 1991; Blain \& Longair 1993). As a result, sub-mm galaxies (hereafter SMGs) turned out to be hundreds of times more numerous than galaxies with similar luminosities in the local universe, suggesting that they constitute the dominant contributor of the CIB and cosmic star formation at $z \gtrsim 1$. This illustrates the very important role that SMGs play in the context of galaxy formation and evolution (see Lonsdale et al. 2006, for a review on (U)LIRGs and SMGs).

One decade after their discovery with $\mathrm{SCUBA}^{1}$ (Smail et al. 1997; Hughes et al. 1998; Barger et al. 1998; Eales et al. 1999), it is generaly acepted that SMGs are heavily dust-obscured

\footnotetext{
1 Submillimeter Common-User Bolometer Array, decommissioned in September 2005 from the James Clerk Maxwell Telescope on Mauna Kea.
} 
galaxies at high redshift $(2<z<3)$ with ULIRG-like luminosities $\left(L_{\mathrm{FIR}} \sim 10^{12} L_{\odot}\right)$ and star formation rates of the order of $1000 M_{\odot} \mathrm{yr}^{-1}$. This enormous bolometric luminosity seems to be dominated by star formation processes induced by galaxy interactions/mergers, although a good fraction $(\sim 30-50 \%)$ of SMGs also host AGN activity (Alexander et al. 2005). First estimates of their physical properties indicate that SMGs are massive, gas rich systems $\left(M_{\text {gas }} \sim 10^{10}-10^{11} M_{\odot}\right)$ in which the starburst region has a typical scale in the range 1-8 kpc (Neri et al. 2003; Chapman et al. 2004; Greve et al. 2005; Tacconi et al. 2006; Wang et al. 2007; Biggs \& Ivison 2008). The available evidence also suggests that SMGs might be the progenitors of massive local ellipticals (Lilly et al. 1999; Smail et al. 2002, 2004; Webb et al. 2003; Genzel et al. 2003; Alexander et al. 2003, 2005; Swinbank et al. 2006).

However, these general properties of SMGs are based on the study of the very brightest examples of this class of object $\left(S_{850 \mu \mathrm{m}} \gtrsim 2 \mathrm{mJy}\right)$, and may not be representative of the entire SMG population. In fact, according to Knudsen et al. (2008), the dominant contribution to the sub-mm extragalactic background comes from the fainter (sub-)mJy sources that cannot usually be detected due to the confusion noise of current instruments $\left(S_{850 \mu \mathrm{m}} \sim 2 \mathrm{mJy}\right)$. The only way in which it has been possible to push bellow this sensitivity limit, is by using the lensing magnification provided by massive clusters of galaxies to increase the effective resolution of SCUBA. This approach has improved the sensitivity of sub-mm maps by factors of a few with respect to blank field surveys, although just a handfull of faint SMGs have being identified so far (Smail et al. 2002; Cowie et al. 2002; Knudsen et al. 2008). Since so little is known about these intrinsically faint sources, it is crucial to further investigate their properties (e.g. spectral energy distributions, morphologies, redshifts, etc.) and assess whether they are different from the observed properties of brighter SMGs.

A promissing strategy to gather information about faint SMGs is to study members that are multiply imaged by clusters of galaxies. In this cases, the magnification factor can go up to 30 (or more), providing not only the opportunity to detect but also to spatially resolve the morphologies and internal dynamics of faint SMGs at a level of detail far greater than would otherwise be possible (see Swinbank et al. 2007, for an example of this technique in the optical). To date, only one multiplyimaged faint SMGs has been confirmed: SMM J16359+6612, located near the core of the cluster A2218 (Kneib et al. 2004, 2005; Garrett et al. 2005; Knudsen et al. 2008, 2009). There are, however, two other clusters which seem to host multiplyimaged SMGs: A1689 (Knudsen et al. 2008) and MS0451.60305 (Chapman et al. 2002; Borys et al. 2004; Berciano Alba et al. 2007).

The case of MS0451.6-0305 is particularly interesting, since the "sub-mm source" (SMM J04542-0301) is an elongated $\left(\sim 1^{\prime}\right)$ region of $850 \mu \mathrm{m}$ emission which is coincident with an optical arc and five NIR sources (see Fig. 1). While the optical arc is the result of a strongly-lensed Lyman Break Galaxy (LBG) at $z_{\text {spect }}=2.911$, a lens model of the cluster predicts that the set of NIR sources could be produced by two triply-imaged EROs ${ }^{2}$ located at almost the same redshift $\left(z_{\text {model }}=2.85 \pm 0.1\right)$. The model also indicates that the ERO pair and the LBG may constitute a merger at $z=2.9$ in the source plane, with their interaction likely being at the origin of the observed sub-mm emission (Borys et al. 2004 , B04 hereafter). Unfortunately, the low resolution $\left(\sim 15^{\prime \prime}\right.$ at $850 \mu \mathrm{m})$ and poor positional accuracy $\left(\sim 2^{\prime \prime}-3^{\prime \prime} \mathrm{rms}\right)$ of SCUBA,

${ }^{2}$ Extremely Red Objects, photometrically defined as $R-K>5.3$.

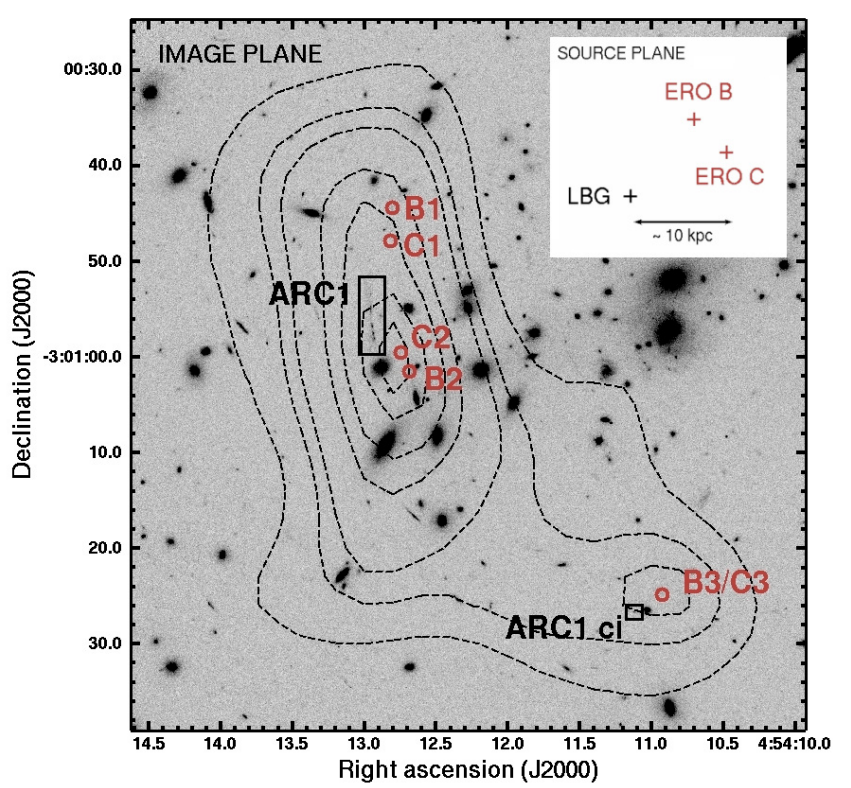

Fig. 1. Summary of the results presented in Borys et al. (2004). SCUBA $850 \mu \mathrm{m}$ contour map of SMM J04542-0301 superimposed upon a HST image of the center of the cluster MS0451.6-0305. Highlighted with rectangles are an optical arc (ARC1) and its counter image (ARC1 ci), produced by a LBG at $z_{\text {spect }}=2.911$. The red circles indicate the positions of five NIR sources that have been interpreted as multiple images produced by two EROs (ERO B, lensed as B1/B2/B3, and ERO C, lensed as $\mathrm{C} 1 / \mathrm{C} 2 / \mathrm{C} 3$ ) at $z_{\text {model }}=2.85 \pm 0.1$. Assuming $z=2.9$ for both the LBG and the EROs, their predicted positions in the source plane would be located withing a region of $\sim 10 \mathrm{kpc}$, suggesting that they constitute a merger.

makes it very difficult to confirm the link between SMM J045420301 and the proposed optical/NIR sources. Moreover, the emission coming from the north-eastern and central regions of the sub-mm emission cannot be reproduced by the arc and the NIR sources (see Fig. 7 in B04), suggesting that it might arise via other sources.

A possible way to overcome this resolution problem is by taking advantage of the observed correlation between the radio synchrotron and FIR emission in star-forming galaxies (van der Kruit 1971; Condon et al. 1982; Helou et al. 1985; Garrett 2002; Appleton et al. 2004; Beelen et al. 2006), which also seems to hold for SMGs out to $z \sim 3$ (Kovács et al. 2006; Vlahakis et al. 2007; Ibar et al. 2008; Michałowski et al. 2009). Thanks to this FIR-radio correlation, radio interferometric observations can be used as a high-resolution proxy for the rest-frame FIR emission observed in the sub-mm. This approach has been extensively used to pinpoint the position of SMGs in order to identify their faint optical counterparts (e.g. Ivison et al. 2000; Barger et al. 2000; Chapman et al. 2005; Ivison et al. 2007).

In Berciano Alba et al. (2007), we reported the detection of $1.4 \mathrm{GHz}$ radio emission coincident with SMM J04542-0301 using VLA ${ }^{3}$ archival data. Part of this radio emission is located in the region between the optical arc and the ERO images, which is consistent with the interacting region of the hypothetical merger being the source of the observed radio and sub-mm emission. We also detected bright radio emission in the central region of SMM J04542-0301, although it is not clear if this

\footnotetext{
3 Very Large Array interferometer of the National Radio Astronomy Observatory (NRAO).
} 
emission is produced by a high- $z$ lensed object or AGN activity associated with a cluster member.

In this paper, we present a higher resolution $1.4 \mathrm{GHz}$ radio map of the center of the cluster MS0451.6-0305 (MS0451 hereafter), obtained after combining the previous VLA archival data (BnA configuration) with new VLA high resolution observations (A configuration). The details of both sets of observations are presented in Sect. 2, together with a description of the data analysis procedure, which has been considerably improved with respect to Berciano Alba et al. (2007). Section 3 is dedicated to the analysis of the final combined data set, from which we characterise the compact and extended radio emission detected within SMM J04542-0301. Identification of optical (HST/ACS $F 814 W$ ) and NIR (SUBARU/CISCO $K^{\prime}$ band) counterparts for the radio detections, as well as their connection with the sub-mm emission, is discussed in Sect. 4. In Sect. 5 we describe a new lens model of the cluster MS0451.6-0305, built to investigate the lensed nature of the compact radio detections. A discussion about the merger scenario proposed by Borys et al. (2004) including the results from the radio observations are presented in Sect. 6. Summary and conclusions are presented in Sect. 7.

The adopted cosmology corresponds to a $\Lambda$ CDM model with $\Omega_{\mathrm{m}}=0.28, \Omega_{\lambda}=0.72$ and $h_{0}=73$ (Spergel et al. 2007).

\section{Radio observations and data reduction}

The $1.4 \mathrm{GHz}$ observations of the cluster MS0451 presented in this paper were obtained with the VLA in A and $\mathrm{B}$ configurations (A-array and B-array hereafter) using the wide-field "pseudocontinuum mode". This mode consists on two IFs of $25 \mathrm{MHz}$, centered at $1.3649 \mathrm{GHz}$ and $1.4351 \mathrm{GHz}$. Each IF contains right and left circular polarizations and it is split into seven $3.125 \mathrm{MHz}$ channels. The absolute flux density scale was set by the flux calibrator $0137+331$ (3C 48), while $0503+020$ was used as the phase calibrator (PHCS hereafter). The data reduction was conducted in classic AIPS using our own semi-automatic pipeline written in Parseltongue (Python interface to classic AIPS, see Kettenis et al. 2006).

\subsection{A-array observations}

The A-array observations consist on $2 \times 6 \mathrm{~h}$ of data acquired the 5th and 10th of February 2006 (correlator integration time of $3.3 \mathrm{~s}$ ). Each IF of each observing day was independently selfcalibrated, resulting in four data sets that were in the end combined to obtain the final A-array map of the target. In order to avoid source smearing, no averaging in frequency nor time was applied to the data. The different steps followed during the data reduction of each epoch are described next.

First, we improved the positional accuracy of the antennas in our data by applying the most recent baseline corrections determined by NRAO. Corrupted data were visually inspected and flagged independently for each channel, IF and polarization at different stages of the data reduction process.

To set the flux scale, amplitude and phase corrections were calculated for $3 \mathrm{C} 48$ using a model of this source provided by AIPS. Then, 3C 48 was used to calibrate the bandpass shape. In the case of the PHCS, we first derived a preliminary calibration in amplitude and phase using a point source model. Subsequent self-calibration produced a more accurate clean component model (CC model hereafter), which was ultimately used to derive the final phase calibration. After proper interpolation, the resultant amplitude and phase solutions were applied to the cluster data, and further refined through self-calibration in order to produce the optimal map for our target.

Figure 2 (top) shows a low resolution $1 \times 1 \mathrm{deg}$ map of the cluster produced with the inner $10 \mathrm{k} \lambda$ baselines of the A-array data. Due to the significant number of bright sources located outside the main lobe of the primary beam (black circle), 37 facets were used for the imaging part of the self-calibration process: 31 overlapping facets that cover the central 0.32 deg radius region, and 6 individual facets centered at the positions of bright NVSS sources ${ }^{4}$ located in an annulus between 0.32 and $0.5 \mathrm{deg}$. radius. The size of each facet is $2048 \times 2048$ pixels, with a pixel scale of $0.247^{\prime \prime}$. Areas for CLEANING were restricted by placing boxes around the brightest sources in each facet. The weighting scheme used for imaging was selected by the IMAGR parameter ROBUST ( $R$ hereafter), with value ranging from +5 (pure natural weighting) to -5 (pure uniform weighting). To produce a good model of the compact emission, $R=0$ was used until the last iteration of the self-calibration procedure, where we switched to $R=5$ to include the extended emission.

From the sources indicated in the top panel of Fig. 2, SRC1 is compact, and it is the brightest and closest to the target, so it should provide the best self-calibration solutions in the target region. However, the extended sources SRCA and SRCB dominate the solutions in the short baselines. In order to get the final calibration only from SRC1 for all baselines, all the sources located outside the central facet were subtracted from the data using the final CC model of the outer 37 facets. Finally, the resultant data set was self-calibrated again using $R=5$ during the imaging process.

This procedure was followed for each IF of each epoch, resulting in four independently calibrated data sets (in which only the sources from the central facet remain) that were concatenated with DBCON to produce the final A-array map of the target.

\subsection{B-array observations}

The B-array observations, made the 9th and 10th of June 2002, were retrieved from the NRAO data archive system $^{5}(7.8 \mathrm{~h}$ in total, correlator integration time of $10 \mathrm{~s}$ ). The first time that these observations were analyzed (Berciano Alba et al. 2007), they were treated as a single epoch and averaged on time, with no independent calibration for each IF. However, since our goal is to combine the data from both array configurations to produce a more sensitive map of the target, the B-array observations were re-reduced following essentially the same procedure described in the previous section. The only difference corresponds to the self-calibration of the cluster data, which we now describe in detail.

Figure 2 (bottom) shows a $1.5 \times 1.5$ deg low resolution map of the cluster produced with the inner $10 \mathrm{~K} \lambda$ baselines of the B-array data. Note that SRC2 is brighter than SRC1, contrary to the situation in the A-array data (top) where SRC2 is resolved. However, since SRC2 is considerably further away from the phase center, the best self-calibration solutions on the target region will still be provided by SRC1. Therefore, the data was self-calibrated in three steps. First, a set of 7 overlapping facets was used to image the central $0.4 \mathrm{deg}$ radius region of the data, while another 36 facets were centered at the positions of NVSS sources located in an annulus between 0.4 and $1.6 \mathrm{deg}$

\footnotetext{
4 The NRAO VLA Sky Survey (NVSS) is a $1.4 \mathrm{GHz}$ continuum survey covering the entire sky north of $-40 \mathrm{deg}$. declination, with a completeness limit about $2.5 \mathrm{mJy}$.
}

5 Project ID AN0109, PI: Nakanishi. 


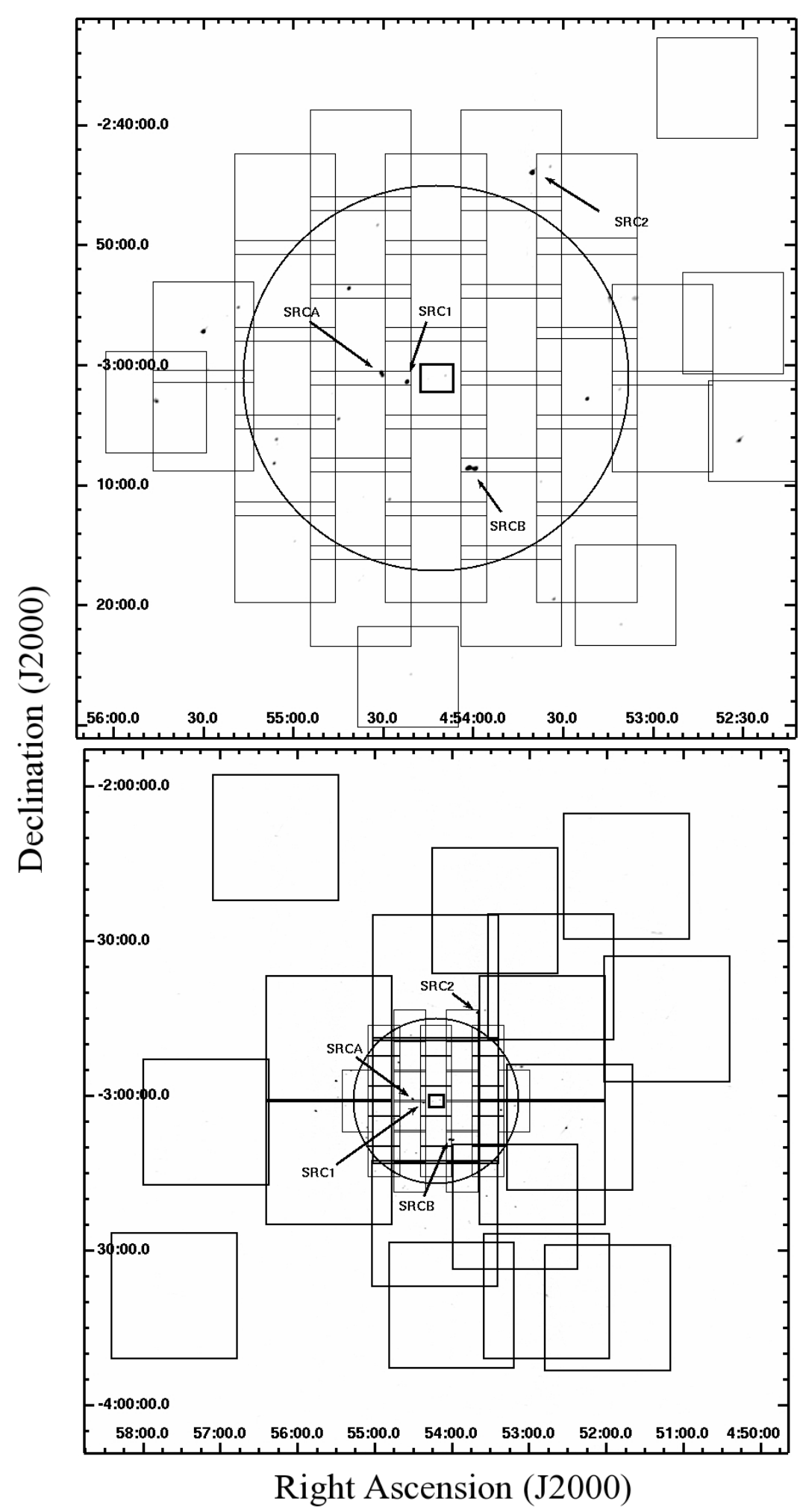

Fig. 2. VLA 1.4 GHz maps centered in the core of the cluster MS0451.6-0305. The maps were produced using the visibilities of the central $10 \mathrm{k} \lambda$ region of the UV-plane only, to enhance the source visibility. The facets used during the selfcalibration of the cluster data are indicted with boxes, while the circle represents the VLA FWHM primary beam $\left(0.5^{\circ}\right.$ at $\left.1.4 \mathrm{GHz}\right)$. The central bold box corresponds to the target region mapped in Fig. 4. The brightest sources in the field are indicated with labels. Top panel: $1^{\circ} \times 1^{\circ}$ map produced with the A-array observations. Each box is $8.4^{\prime} \times 8.4^{\prime}$. Bottom panel: $1.5^{\circ} \times 1.5^{\circ}$ map produced with the B-array observations. The big boxes (used in the first step of the selfcalibration procedure) are $27.8^{\prime} \times 27.8^{\prime}$ in size, whereas the small boxes (used in the second step) are $6.8^{\prime} \times 6.8^{\prime}$.

radius (big boxes in the bottom panel of Fig. 2). Each facet is $2048 \times 2048$ pixels, with a pixels size of $0.814^{\prime \prime}$. Once the self-calibration procedure was completed, the $\mathrm{CC}$ model from the 42 outer facets was subtracted from the data, leaving only the sources located in the central facet. In the second step, the central region of the resultant data set is imaged again using 


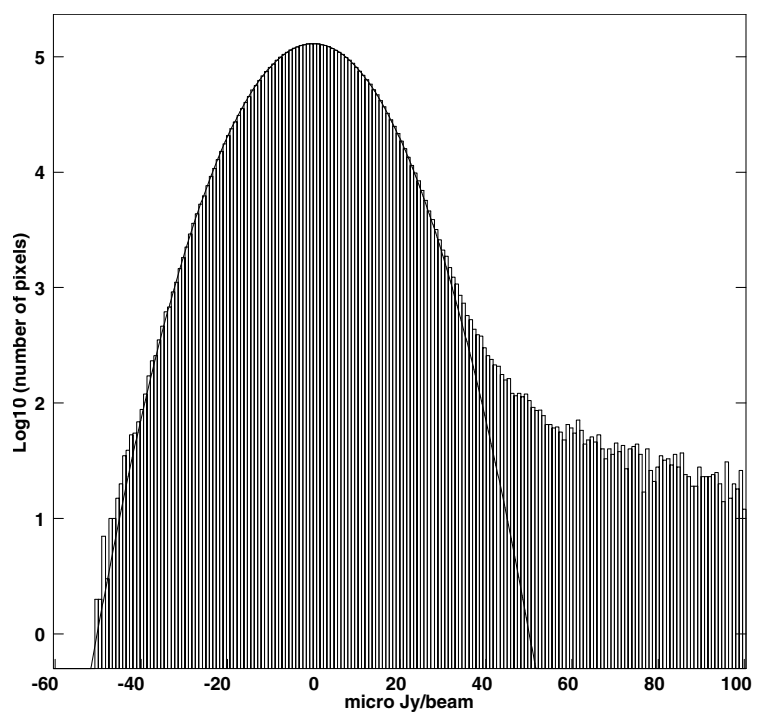

Fig. 3. Histogram of the $\mathrm{A}+\mathrm{B}$ array clean map produced using $R=0.6$. The black curve is the best Gaussian fit to the noise peak, while the positive excess on the right side is due to source emission. The good agreement between the fit and the data in the negative side of the Gaussian indicates that the noise of the map is well behaved, making faint detections more reliable.

31 overlapping facets of $500 \times 500$ pixels. After following the same self-calibration procedure used in the first step, the best $\mathrm{CC}$ model of the outer 30 facets was subtracted from the data. In the third step, this final data set is calibrated (phase only) using a solution interval of $5 \mathrm{~min}$ and $R=5$.

As in the case of the A-array observations, the four independently calibrated data sets obtained in this way (one per epoch and IF) were then combined and imaged to produce the final B-array map of the target.

\section{Analysis of the A+B-array map}

Before the A-array and B-array observations could be combined to produce the final radio map of the cluster center, it was necessary to correct for a single position shift between the two calibrated data-sets. This correction was achieved by performing a global phase-selfcalibration of the B-array data set using the best A-array CC model.

To produce the map with the best compromise between sensitivity and noise properties, we explored the full range of values available for the IMAGR parameter ROBUST. The histograms of the resultant clean maps show that the noise distribution is closest to Gaussian when $R=0.6$ (see Fig. 3). Note also that the rms noise of the $R=0.6$ map $(\sim 10 \mu \mathrm{Jy})$ is only $2 \%$ higher than the one obtained for $R=5$, which is the most sensitive map that can be produced with this data.

\subsection{Catalog of radio sources}

Figure 4 shows a detail on the $R=0.6$ map of the cluster center produced by cleaning the data down to a depth of $5.5 \mu \mathrm{Jy}$. The contours indicate the region of extended sub-mm emission reported by $\mathrm{B} 04$, in which six of the eight identified radio sources are located. Postage stamps of these six sources and their CC models are shown in Fig. 5.

The radio detections located outside the sub-mm emission, labeled $\mathrm{Fd}$ and $\mathrm{Fe}$, are the same sources reported in Berciano Alba et al. (2007). Their morphology in the new high resolution radio map is very elongated, typical of radio jets produced by AGNs. In the case of the other 6 sources, the morphology and distribution of their clean components show that, with the exception of the (almost) point-like structure of E1, the rest of the sources are extended. In particular, RJ shows a bright elongated main body and an irregular $4 \sigma$ extension towards the west. Note however, that the amorphous extensions present in the $2 \sigma$ contours of E1, CR2 and E3 are not considered to be part of their real structure. In the following, references to the $2 \sigma$ contours of these sources will implicitly exclude these amorphous extensions.

The peak positions and peak fluxes of all the radio detections were calculated with the task MAXFIT, which fits a quadratic function to a rectangular area that encloses the source (in our case, a rectangle delimited by the $2 \sigma$ contours of the source). The total flux, on the other hand, was determined in two different ways: (i) by integrating the emission over the area defined by the $2 \sigma$ contour of the source $\left(S_{2 \sigma}\right)$; and (ii) by adding the flux of all the clean components of the source model $\left(S_{\mathrm{CC}}\right)$. In this way, we can calculate the flux excess contained in the clean component model respect to the $2 \sigma$ flux, which is an estimate of the amount of flux contained in the wings of the PSF-convolved sources below the $2 \sigma$ contour.

The results obtained for the different parameters are listed in Table 1. Note that, since the cleaning procedure is stopped at certain flux level (half a sigma below the noise level), it is expected to have some left-over emission from the sources in the residual map, specially in the case of extended sources. To estimate this left-over flux, we have integrated the emission inside the $2 \sigma$ contours of each source in the residual map (see $S_{\text {resid }}$ in Table 1). The results show that the $S_{\mathrm{CC}}$ for CR1 and RJ is underestimated by $\sim 6 \%$, and by $\sim 10 \%$ in the case of E2 and E3. The negative values for $S_{\text {resid }}$ obtained for E1 and CR2 indicate that all the flux has been included in the CC model.

We also tried to characterize the properties of the six sources located within the sub-mm emission region by comparing them with Gaussians. The task JMFIT was used to fit simultaneously all the parameters of an elliptical Gaussian in the same rectangular area used with MAXFIT. Since the shape of RJ is far from being elliptical, we restricted the Gaussian fitting to the area that only contains the $2 \sigma$ contours of the bright main body. The center and FWHM of the best fitting Gaussians are shown in Fig. 5.

The parameters derived from the best fits and their formal errors are listed in Table 2, where the sum of the square of the residuals is a measure of the relative goodness of the fit. As expected, the worst fits are obtained for RJ and E3, since they have the most irregular shapes. The best fits correspond to CR 1 and E2, but note that the FWHM of the Gaussian in CR1 does not include the north-east $2 \sigma$ extension.

When compared with Table 1, we see that the Gaussian fit provides basically the same estimates for the position (RA, Dec) and flux peak $\left(S_{\mathrm{Pk}}\right)$. The nominal integrated flux $\left(S_{\mathrm{Gauss}}\right)$ is in general larger than $S_{\mathrm{CC}}$ (except for E1), but consistent within the errors. The largest discrepancies are found for E3 and CR2 (see $\frac{S_{\mathrm{CC}}-S_{\text {Gauss }}}{S_{\text {Gauss }}}$ in Table 1 ), probably because $S_{\text {Gauss }}$ is including some flux from the $2 \sigma$ amorphous extensions in these two sources that were not accounted for in the clean component model.

Finally, we would like to emphasize that, despite the faintness of CR1 and CR2 (SNR 4.6), the histogram presented in Fig. 3 and the distribution of $-4 \sigma$ peaks in the final map (only a small number, located far away from the target region) suggests that they are real detections. 


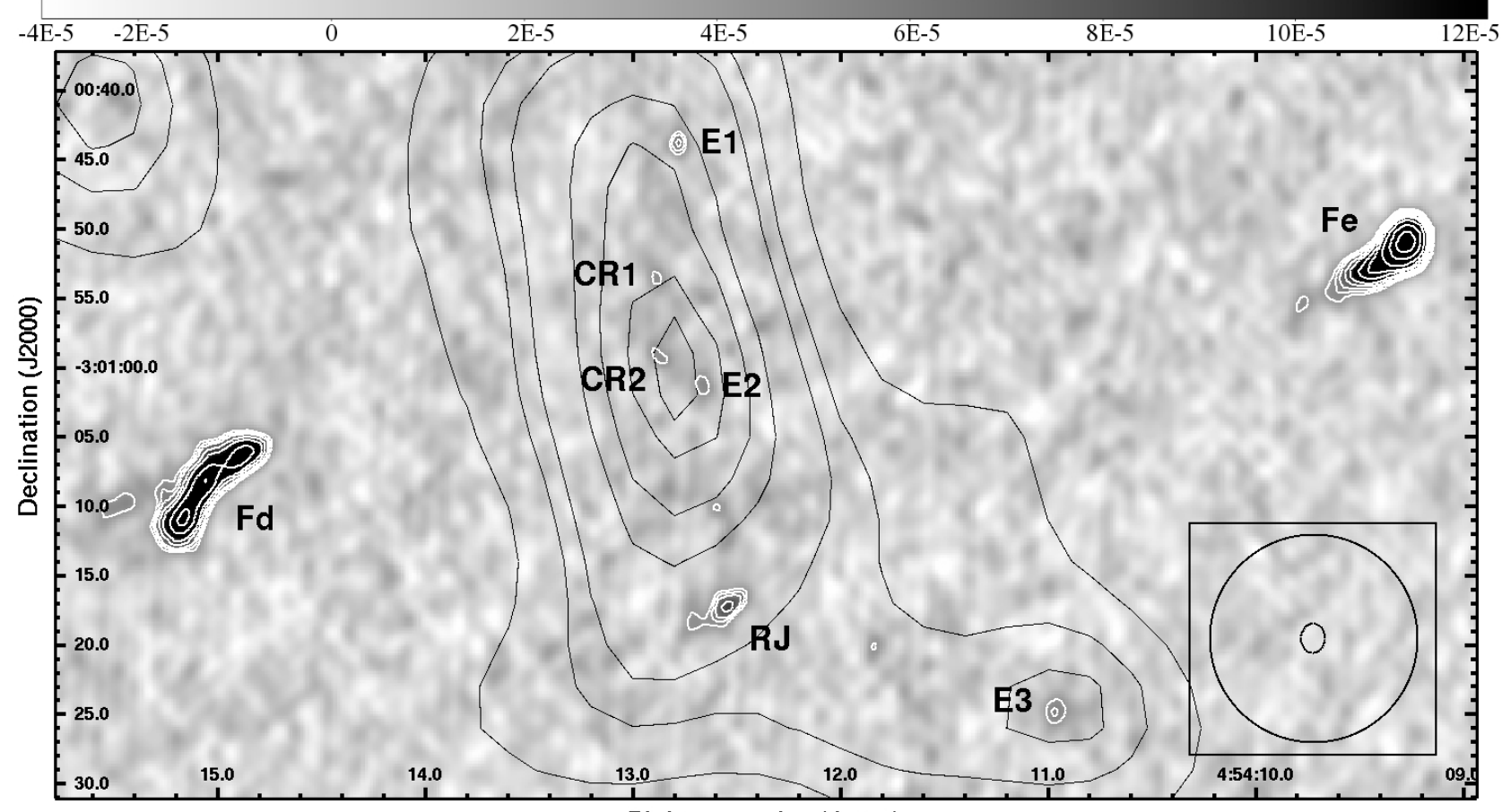

Right ascension (J2000)

Fig. 4. VLA $1.4 \mathrm{GHz}$ map derived from the $\mathrm{A}+\mathrm{B}$-array data set using $R=0.6$. The greyscale has units of Jy beam ${ }^{-1}$, and the corresponding contours (white) are drawn at $-4,4,5,6,8,10,14,18,28,48$ and 78 times the $1 \sigma$ noise level of $10.34 \mu \mathrm{Jy}_{\text {beam }}{ }^{-1}$. The black lines $(4,6,7,9,10$, 11 and $11.5 \mathrm{mJy}_{\text {beam }}{ }^{-1}$ ) correspond to the SCUBA $850 \mu \mathrm{m}$ contour map published in Borys et al. (2004). A comparison of the beam size of both contour maps is shown in the bottom right corner: $2.14^{\prime \prime} \times 1.71^{\prime \prime}$ at a position angle of $-1^{\circ} .43$ for the radio map, and $15^{\prime \prime} \times 15^{\prime \prime}$ for the submm map.
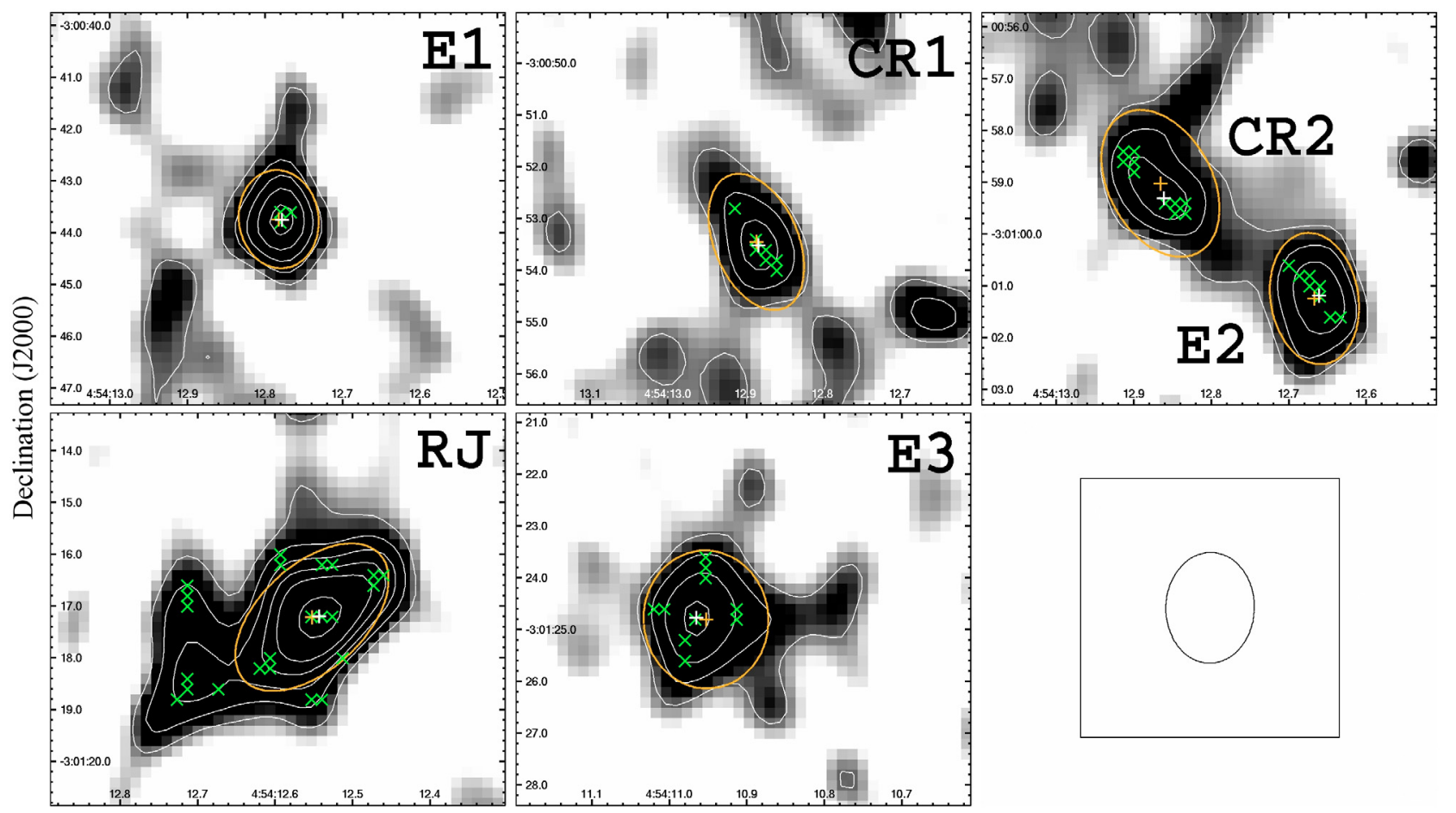

Right ascension (J2000)

Fig. 5. Detail of the six radio sources located within the sub-mm emission region in Fig. 4 . The white radio contours are drawn at 2, $3,4,5,6$ and 8 times the $1 \sigma$ noise level of $10.34 \mu \mathrm{Jy}_{\text {beam }}{ }^{-1}$. The radio peak positions $\left(S_{\mathrm{Pk}}\right)$ listed in Table 1 are indicated with white plus-symbols. The green crosses correspond to the positions of the clean components in each source model. Orange plus-symbols and ellipses represent the center and $F W H M$ of the best fitting Gaussian for each source. A scaled version of the radio beam $\left(2.14^{\prime \prime} \times 1.71^{\prime \prime}\right.$ at a position angle of -1.43$)$ is included in the bottom right panel. 
Table 1. Information about the identifyed radio sources in the core of MS0451.

\begin{tabular}{|c|c|c|c|c|c|c|c|c|c|}
\hline Name & $\begin{array}{c}\mathrm{RA}\left(+4^{\mathrm{h}} 54^{\mathrm{m}}\right) \\
\mathrm{J} 2000(\mathrm{~s})\end{array}$ & $\begin{array}{l}\operatorname{Dec}\left(-3^{\circ}\right) \\
J 2000\left({ }^{\prime},{ }^{\prime \prime}\right)\end{array}$ & $\begin{array}{c}S_{\mathrm{Pk}} \\
\left(\mu \mathrm{Jy} \mathrm{beam}^{-1}\right)\end{array}$ & $\begin{array}{l}S_{2 \sigma} \\
(\mu \mathrm{Jy})\end{array}$ & $\begin{array}{l}S_{\mathrm{CC}} \\
(\mu \mathrm{Jy})\end{array}$ & $\begin{array}{l}S_{\text {resid }} \\
(\mu \mathrm{Jy})\end{array}$ & $\begin{array}{c}\frac{S_{\mathrm{CC}}-S_{2 \sigma}}{S_{2 \sigma}} \\
(\%)\end{array}$ & $\begin{array}{c}\frac{S_{\mathrm{CC}}-S_{\text {Gauss }}}{S_{\text {Gauss }}} \\
(\%)\end{array}$ & SNR \\
\hline E1 & $12.78 \pm 0.01$ & $00: 43.8 \pm 0.2$ & $66 \pm 10$ & $42.5 \pm 12$ & 61.6 & -1.4 & 45 & 17 & 6.4 \\
\hline CR1 & $12.88 \pm 0.01$ & $00: 53.5 \pm 0.2$ & $47 \pm 10$ & $36.6 \pm 12$ & 47.2 & 2.9 & 29 & -16 & 4.6 \\
\hline CR2 & $12.86 \pm 0.01$ & $00: 59.3 \pm 0.2$ & $47 \pm 10$ & $43.8 \pm 12$ & 60.1 & -0.1 & 37 & -21 & 4.6 \\
\hline E2 & $12.66 \pm 0.01$ & $01: 01.2 \pm 0.2$ & $51 \pm 10$ & $43.2 \pm 12$ & 52.0 & 5.1 & 20 & -15 & 5.0 \\
\hline RJ bright & $12.54 \pm 0.01$ & $01: 17.2 \pm 0.1$ & $92 \pm 10$ & $121.2 \pm 17$ & 135.9 & - & 12 & -17 & 8.9 \\
\hline RJ & $12.54 \pm 0.01$ & $01: 17.2 \pm 0.1$ & $92 \pm 10$ & $168.0 \pm 22$ & 195.3 & 10.5 & 16 & - & 8.9 \\
\hline E3 & $10.97 \pm 0.01$ & $01: 24.8 \pm 0.2$ & $54 \pm 10$ & $63.3 \pm 15$ & 66.8 & 6.5 & 5 & -28 & 5.2 \\
\hline $\mathrm{Fd}$ & $15.162 \pm 0.003$ & $01: 10.83 \pm 0.05$ & $199 \pm 10$ & $1180.9 \pm 42$ & 1260 & 29.5 & 7 & - & 19.2 \\
\hline $\mathrm{Fe}$ & $09.2733 \pm 0.0007$ & $00: 50.90 \pm 0.01$ & $1068 \pm 10$ & $1769.2 \pm 34$ & 1870 & 16.3 & 6 & - & 103.3 \\
\hline
\end{tabular}

The columns show: position (RA, Dec), peak flux density $\left(S_{\mathrm{Pk}}\right)$, total flux density inside the $2 \sigma$ contour $\left(S_{2 \sigma}\right)$, total flux density obtained by adding the flux from all the clean components used to model the source $\left(S_{\mathrm{CC}}\right)$, total flux density inside the $2 \sigma$ contour in the residual map $\left(S_{\text {resid }}\right)$, comparison between the total flux derived from the CC model and the $2 \sigma$ contours $\left(\frac{S_{\mathrm{CC}}-S_{2 \sigma}}{S_{2 \sigma}}\right)$, comparison between the total flux derived from the CC model and the Gaussian fit $\left(\frac{S_{\mathrm{CC}}-S_{\text {Gauss }}}{S_{\text {Gauss }}}\right)$ and signal-to-noise ratio (SNR). The quoted errors in RA and DEC correspond to the expected positional uncertainty of a point-like source in the map $(F W H M / 2 \times S N R$, were $F W H M$ is the full with half maximum of the clean beam), while the error in the flux peak is estimated to be the same as the rms noise of the map. The error in the $2 \sigma$ flux was calculated as rms $\times \sqrt{N}$, where rms is the rms noise of the map, and $N$ is the number of beams within the area delimited by the $2 \sigma$ contour of the source. The raw "RJ bright" lists the fluxes for RJ estimated withouth taking into account the $4 \sigma$ west extension. Note that the positions listed here were derived before aligning the radio map respect to the HST image.

Table 2. Gaussian fitting results for the identified compact radio sources in the core of MS0451.

\begin{tabular}{lcccccccc}
\hline \hline Name & $\begin{array}{c}\text { RA }\left(+4^{\mathrm{h}} 54^{\mathrm{m}}\right) \\
\mathrm{J} 2000(\mathrm{sec})\end{array}$ & $\begin{array}{c}\text { Dec }\left(-3^{\circ}\right) \\
\mathrm{J} 2000\left({ }^{\prime},{ }^{\prime \prime}\right)\end{array}$ & $\begin{array}{c}S_{\text {Pk }} \\
\left(\mu \mathrm{Jy} \text { beam }^{-1}\right)\end{array}$ & $\begin{array}{c}S_{\text {Gauss }} \\
(\mu \mathrm{Jy})\end{array}$ & $\begin{array}{c}\text { Maj Axis } \\
(\operatorname{arcsec})\end{array}$ & $\begin{array}{c}\text { Min Axis } \\
(\operatorname{arcsec})\end{array}$ & $\begin{array}{c}\text { PA } \\
(\mathrm{deg})\end{array}$ & $\begin{array}{c}\text { SSR } \\
\left(\times 10^{-9} \mathrm{Jy}^{2} \text { beam }^{-2}\right)\end{array}$ \\
\hline E1 & $12.782 \pm 0.007$ & $00: 43.7 \pm 0.1$ & $66.1 \pm 10.3$ & $53.0 \pm 15.5$ & $0.00 \pm 1.14$ & - & - & 2.2 \\
CR1 & $12.89 \pm 0.01$ & $00: 53.5 \pm 0.3$ & $45.6 \pm 10.2$ & $56.2 \pm 20.4$ & $1.87 \pm 1.87$ & $0.00 \pm 1.17$ & $31.4 \pm 44.7$ & 0.6 \\
CR2 & $12.87 \pm 0.01$ & $00: 59.0 \pm 0.3$ & $45.9 \pm 10.0$ & $76.3 \pm 24.5$ & $2.28 \pm 1.22$ & $0.73 \pm 0.99$ & $37.6 \pm 3.8$ & 1.8 \\
E2 & $12.67 \pm 0.01$ & $01: 01.2 \pm 0.2$ & $51.6 \pm 10.3$ & $61.2 \pm 20.0$ & $1.44 \pm 1.44$ & $0.00 \pm 1.07$ & - & 0.9 \\
RJ bright & $12.55 \pm 0.01$ & $01: 17.2 \pm 0.1$ & $82.9 \pm 9.8$ & $164 \pm 27.6$ & $3.02 \pm 0.56$ & $0.51 \pm 0.73$ & $128 \pm 10.9$ & 12.8 \\
E3 & $10.95 \pm 0.01$ & $01: 24.8 \pm 0.2$ & $52.6 \pm 9.9$ & $92.5 \pm 25.5$ & $1.71 \pm 0.95$ & $1.58 \pm 1.58$ & $70.5 \pm 39.0$ & 3.4 \\
\hline
\end{tabular}

The columns show: position (RA, Dec), peak flux density $\left(S_{\mathrm{Pk}}\right)$, total flux density $\left(S_{\text {Gauss }}\right)$, deconvolved Gaussian sizes (major axis, minor axis and position angle) with their corresponding formal errors, and the sum of the square of the residuals reported from the fit (SSR). Cases where the parameters of the Gaussian fits are not well constrained are indicated by a dash. Note that the positions listed here were derived before aligning the radio map respect to the HST image.

\subsection{Disentangling the compact and extended radio emission}

As can be seen in Fig. 6, the region between E1 and E2 shows an enhanced low level background when compared with the rest of the map, suggesting the presence of diffuse radio emission that has been partially resolved out. From now on, we will refer to this diffuse emission as the extended component, whereas E1, E2, E3, CR1 and CR2 will be consider compact sources by comparison.

If present, this extended component would only be detectable on short baselines, while the emission produced by compact sources will be picked up by all baselines at different resolution levels. Therefore, a map produced using only the short baselines does not allow us to distinguish between extended emission and a compact source observed at low resolution. The only way to isolate the extended component is by subtracting a model of the compact sources from the data.

Given the faintness of CR1 and CR2, and the complicated low level background in this region, the choice of boxes during the cleaning procedure becomes a highly subjective issue, and even the decision of cleaning CR1 and CR2 (and hence their $\mathrm{CC}$ models) can be questionable. For this reason, we produced a new version of the map presented in Fig. 4 (following the same procedure) in which CR1 and CR2 have not been cleaned. Then, we calculated $S_{\mathrm{Pk}}, S_{2 \sigma}$ and $S_{\mathrm{CC}}$ for all the sources in the new map, and compared them with the values presented in Table 1. In the case of E2 (which is the closest source to CR1 and CR2) we find differences of $\sim 10 \%$ for $S_{\mathrm{CC}}$ and $\sim 14 \%$ for $S_{2 \sigma}$, whereas for the rest of the sources the differences in all three parameters are less than $7 \%$. Since these variations are well withing the errors estimated for $S_{\mathrm{Pk}}$ and $S_{2 \sigma}$ in Table 1, we can use the CC models of CR1 and CR2 presented in Fig. 5 without compromising the results.

Figure 7 shows three $\sim 5^{\prime \prime}$ resolution maps of the region between E1 and E2, produced by tapering the data with a Gaussian of $F H W M=30 \mathrm{k} \lambda$. In map (A) no compact sources were subtracted before the tapering and imaging. The central map (B) shows the result of subtracting the CC model of E1 and E2 from the data, and map (C) shows what is left after subtracting all the compact sources. Given the robust detection of a $6.6 \sigma$ elongated source in panel (B), the presence of resolved extended radio emission in this region of the $\sim 2^{\prime \prime}$ map (Fig. 6) is confirmed. Using the $2 \sigma$ contour of map (B) as template, the integrated fluxes in panels (B) and (C) are $S_{\mathrm{B}}=173.52 \pm \mu \mathrm{Jy}$ and $S_{\mathrm{C}}=84.14 \pm \mu \mathrm{Jy}$. The flux error was calculated as $\mathrm{rms} \times \sqrt{N}$, where $r m s$ is the rms noise of the map, and $N$ is the number of 


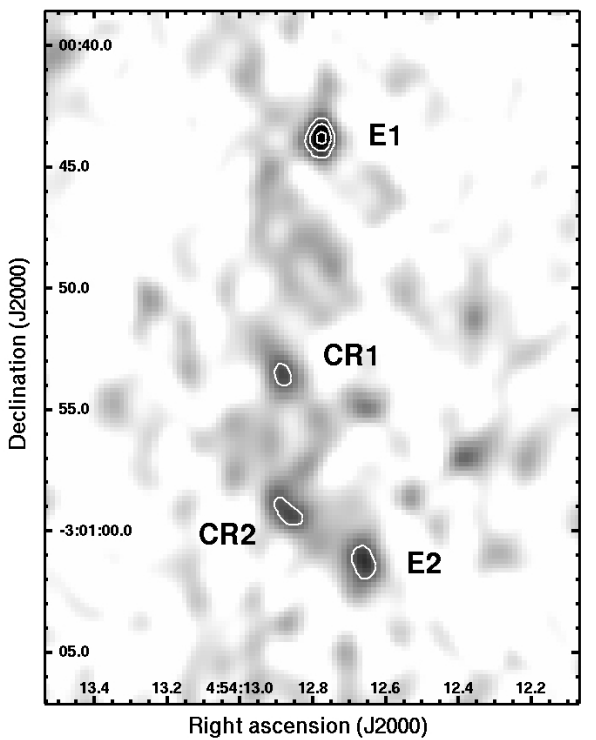

Fig. 6. Detail of Fig. 4. The grey scale has been modified to make more evident the low level background in this region of the radio map.

beams within the area delimited by the $2 \sigma$ contour of map (B). These numbers indicate that $\sim 50 \%$ of the flux in panel (B) has been included in the $\sim 2$ " CC model of CR1 and CR2. Therefore, it seems that CR1 and CR2 constitute two relatively compact regions of an extended radio source located between E1 and E2. However, given the faintness of CR1, CR2 and the extended component in panel $(\mathrm{C})$, deeper observations will be required to confirm this result.

Finally, to estimate the relative contribution of all the compact sources respect to the extended emission, we used the $2 \sigma$ contour of map (A) as template to calculate the integrated flux of each map in Fig. 7: $S_{\mathrm{A}}=285 \pm 33 \mu \mathrm{Jy}, S_{\mathrm{B}}=204 \pm 33 \mu \mathrm{Jy}$ and $S_{\mathrm{C}}=109 \pm 32 \mu \mathrm{Jy}$. The comparison of these fluxes indicates that $38 \%$ of the emission comes from an extended component, whereas $62 \%$ is produced by the compact sources (28\% from $\mathrm{E} 1$ and E2, 34\% from CR1 and CR2). As it will be discussed in detail in Sect. 4.1, the presence of this extended radio component constitutes a strong observational evidence in favor of the merger scenario proposed in B04.

\section{Multiwavelength counterparts}

Table 3 summarizes the relevant information about all the multiwavelength data of MS0451 (optical, NIR and sub-mm) that we have collected from the literature to compare with our radio map. To identify possible counterparts of the radio sources, the radio and $K^{\prime}$ band observations were aligned with respect to the HST image ${ }^{6}$ using the IRAF package CCMAP. For the alignment of the radio map we used 13 compact radio sources with reliable bright counterparts in the HST image, whereas 93 compact NIR sources were used to align the $K^{\prime}$ band image. We find an rms scatter of $0.29^{\prime \prime}$ between the radio map and the HST image, and $0.06^{\prime \prime}$ between the $K^{\prime}$ band and the HST images. Therefore, the inferred error in the alignment is $0.29 / \sqrt{13}=0.08^{\prime \prime}$ for the radio map and $0.06 / \sqrt{93}=0.006^{\prime \prime}$ for the $K^{\prime}$ band image. These errors are negligible compared with the (relative) astrometric

\footnotetext{
6 Astrometry matched to Subaru/Suprime-Cam observations of MS0451 for the PISCES survey (Kodama et al. 2005).
}

accuracy of the sources in each map, so they will be ignored in further analysis.

\subsection{Comparison between radio and optical/NIR}

Figures 8, 9 and 14 show different details of the HST image where the radio detections are located. The positions of the relevant sources are indicated with circles: blue for the radio sources and red for the $K^{\prime}$ band sources. The counterparts of the sub$\mathrm{mm}$ emission proposed by B04 have been indicated following the nomenclature introduced in Fig. 1. Note that the images B3 and $\mathrm{C} 3$ are supposed to be unresolved in the $K^{\prime}$ band image presented in B04, and therefore referred to as B3/C3.

The relative position of the radio and NIR sources in these figures suggests that E1, E2 and E3 might be associated with $\mathrm{B} 1, \mathrm{~B} 2$ and B3/C3. To better quantify these associations, as well as identify other possible counterparts, we have used Bayesian inference to calculate the probability that the compact radio sources are physically associated with their nearest optical/NIR sources. The mathematical expression of this probability has been derived as follows.

Lets $P(\mu \mid d)$ be the probability density of the true distance $\mu$ between two sources given an observed distance $d$. Following Bayesian inference, this probability is given by:

$P(\mu \mid d)=\frac{L(d \mid \mu) P(\mu)}{P(d)}$

where $L(d \mid \mu)$ is the likelihood of the observed distance given a true distance, $P(\mu)$ is the probability density of the true distance before any measurement, and $P(d)$ is the probability density of the observed distance for all possible distances (a normalization constant).

As pointed out in Churchman et al. (2006), the likelihood of the observed distance between two sources which positions are described by a Gaussian distribution is not Gaussian. Instead, this likelihood in two dimensions is given by:

$L(d \mid \mu)=\frac{\mathrm{d} I_{0}\left(\mathrm{~d} \mu /\left(\sigma_{1}^{2}+\sigma_{2}^{2}\right)\right)}{\sigma_{1}^{2}+\sigma_{2}^{2}} \exp \left(\frac{-\mu^{2}-d^{2}}{2\left(\sigma_{1}^{2}+\sigma_{2}^{2}\right)}\right)$.

Where $\sigma_{1}$ and $\sigma_{2}$ are the errors in the position of each source, and $I_{0}$ is the modified Bessel function of integer order zero.

Since the area inside an annulus at radius $\mu$ increases as $\mu$ $\mathrm{d} \mu$, we assume that, for a source located at a random position, $P(\mu)=\mu$. Therefore:

$P(\mu \mid d) \propto L(d \mid \mu) \cdot \mu$

Which normalized gives:

$B(\mu \mid d)=\frac{L(d \mid \mu) \cdot \mu}{\int_{0}^{\infty} L(d \mid \mu) \mathrm{d} \mu}$.

The integral of this function between $\mu_{1}$ and $\mu_{2}$ gives the probability that, for a given pair of source centroids with observed offset $d$, the true distance between them lies between $\mu_{1}$ and $\mu_{2}$. Intuitively, one would say that the condition for two sources to be counterparts of each other is $\mu=0$. The problem is that, in the case of extended sources like galaxies, the peak of emission at different wavelenghts can be located at different positions, which means that $\mu \neq 0$ despite the fact that they are physical counterparts. For this reason, we will consider that a radio source has an 


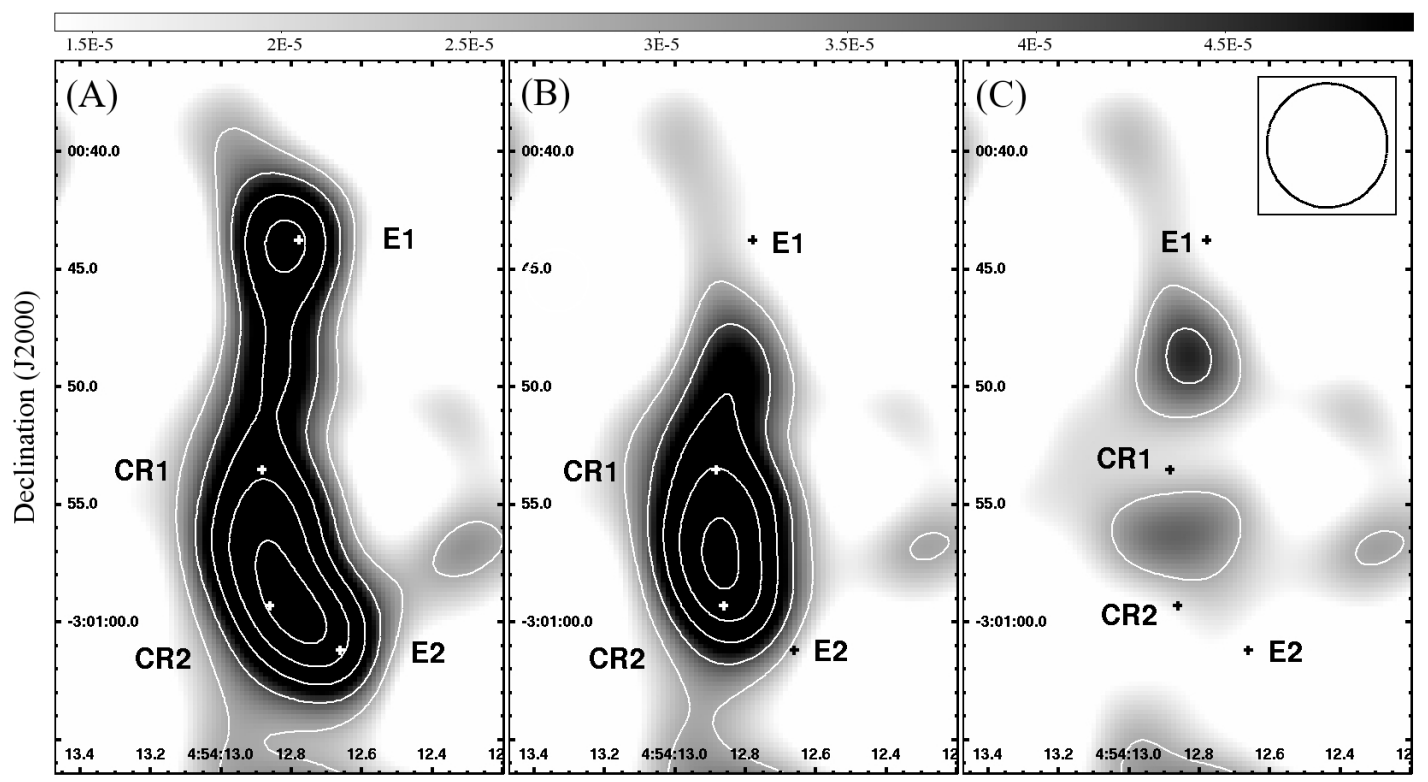

Right ascension (J2000)

Fig. 7. VLA $1.4 \mathrm{GHz}$ maps obtained by tapering the A+B-array data set with a Gaussian of $F W H M=30 \mathrm{k}$. The crosses indicate the positions of the radio detections identified in Fig. 4. Panel (A) includes all the emission between E1 and E2. In panel (B) the CC models of E1 and E2 were subtracted from the data before the tapering and cleaning procedure. Panel (C) shows what is left when CR1 and CR2 are also subtracted from the data. The gray-scale has units of Jy beam ${ }^{-1}$, and the corresponding contours are drawn at 2, 3, 4, 5 and 6 times the $1 \sigma$ noise level of $14 \mu \mathrm{Jy}_{\text {beam }}{ }^{-1}$. The dimensions of the beam are 5.27" $\times 5.12^{\prime \prime}$ at a position angle of $-88^{\circ} .25$ (panel (C), top right corner).

Table 3. Observations details.

\begin{tabular}{|c|c|c|c|c|c|c|}
\hline Instrument & $\begin{array}{c}\text { Frequency (filter) } \\
(\mu \mathrm{m})\end{array}$ & $\begin{array}{c}\begin{array}{c}\text { Observing dates } \\
(\mathrm{dd} / \mathrm{mm} / \mathrm{yy})\end{array} \\
\end{array}$ & $\begin{array}{c}\text { Total exposure time } \\
(\mathrm{ks})\end{array}$ & $\begin{array}{l}\begin{array}{l}\text { Depth } \\
\text { (mag) }\end{array} \\
\end{array}$ & $\begin{array}{c}\begin{array}{c}\text { Resolution } \\
(\operatorname{arcsec})\end{array} \\
\end{array}$ & $\overline{\text { References }}$ \\
\hline HST/ACS & $0.83(F 814 W)$ & - & 2.4-2.7 per pointing & 25 & 1.0 & $\begin{array}{l}\text { Moran et al. (2007) } \\
\text { Smith et al., in prep. }\end{array}$ \\
\hline SUBARU/CISCO & $2.13\left(K^{\prime}\right)$ & $17,18 / 11 / 2000$ & 9.22 & 22.1 & $0.6-0.8$ & Takata et al. (2003) \\
\hline JCMT/SCUBA & 850 & 03/09/1998 & 22 & - & 15.0 & Borys et al. (2004) \\
\hline
\end{tabular}

${ }^{1}$ Data products from their wide field survey in MS0451 are available at http://www. astro. caltech. edu/ smm/clusters/. Further information on the data and survey can be found in Chap. 2 of the Ph.D. Thesis of Sean Moran.

optical/NIR counterpart when the centroid of the source emission lies inside the area of the optical/NIR source. The probability of meeting this condition is given by:

$P_{\text {assoc. }}=\int_{0}^{r_{\text {src }}} B(\mu \mid d) \mathrm{d} \mu$.

A list with the nearest optical/NIR sources of each radio detection, together with their associated probabilities, is presented in Table 4. As expected, only the images of ERO B have a nonnegligible probability of being associated with some of the radio sources (E1, E2 and E3). However, if E1, E2 and E3 are indeed the radio counterparts of $\mathrm{B} 1, \mathrm{~B} 2$ and $\mathrm{B} 3$, they have to be multiple images produced by a radio source at $z \sim 2.9$. A quantitative analysis of this lensing scenario is presented in Sect. 5.4.

Finally, note that no optical/NIR counterpart has been identified with RJ. Therefore, given the depths of the optical and NIR images, it seems more likely that the extended morphology of RJ corresponds to an AGN rather than a resolved low redshift star forming galaxy. Within the AGN scenario, we expect that the peak of the radio emission corresponds to the position of an undetected optical source, and the extensions in opposite directions are two jets coming from it. Another conceivable scenario would be that the galaxy G14 is an AGN host with a one sided radio extension. However, the mayor axes of RJ does not seem to be aligned with $\mathrm{G} 14$, and this kind of AGN is not very common.

\subsection{Comparison between radio and sub- $\mathrm{mm}$}

In this section, we will use the FIR-radio correlation to check whether the radio detections could be associated with the observed sub-mm emission. To make this kind of analysis, it is necessary to match the resolutions of the radio and sub-mm observations. Therefore, the $\mathrm{A}+\mathrm{B}$ array data was tapered with a Gaussian of $8.5 \mathrm{~K} \lambda^{7}$ and restored with a clean beam of $15^{\prime \prime} \times 15^{\prime \prime}$ at the end of the imaging process. The left panel of Fig. 10 shows the final tapered radio map (white contours) superimposed upon the sub-mm map (black contours) and the HST image of the cluster core. The positions of the detections in the high resolution radio map have been indicated with crosses as reference.

If all the observed sub-mm emission would be produced by a single SMG that does not host an AGN, it is expected that the radio and sub-mm morphologies would resemble each other. This is because the origin of the FIR-radio correlation seems to

7 Which corresponds to a clean beam of $15.78^{\prime \prime} \times 14.65^{\prime \prime}$. 


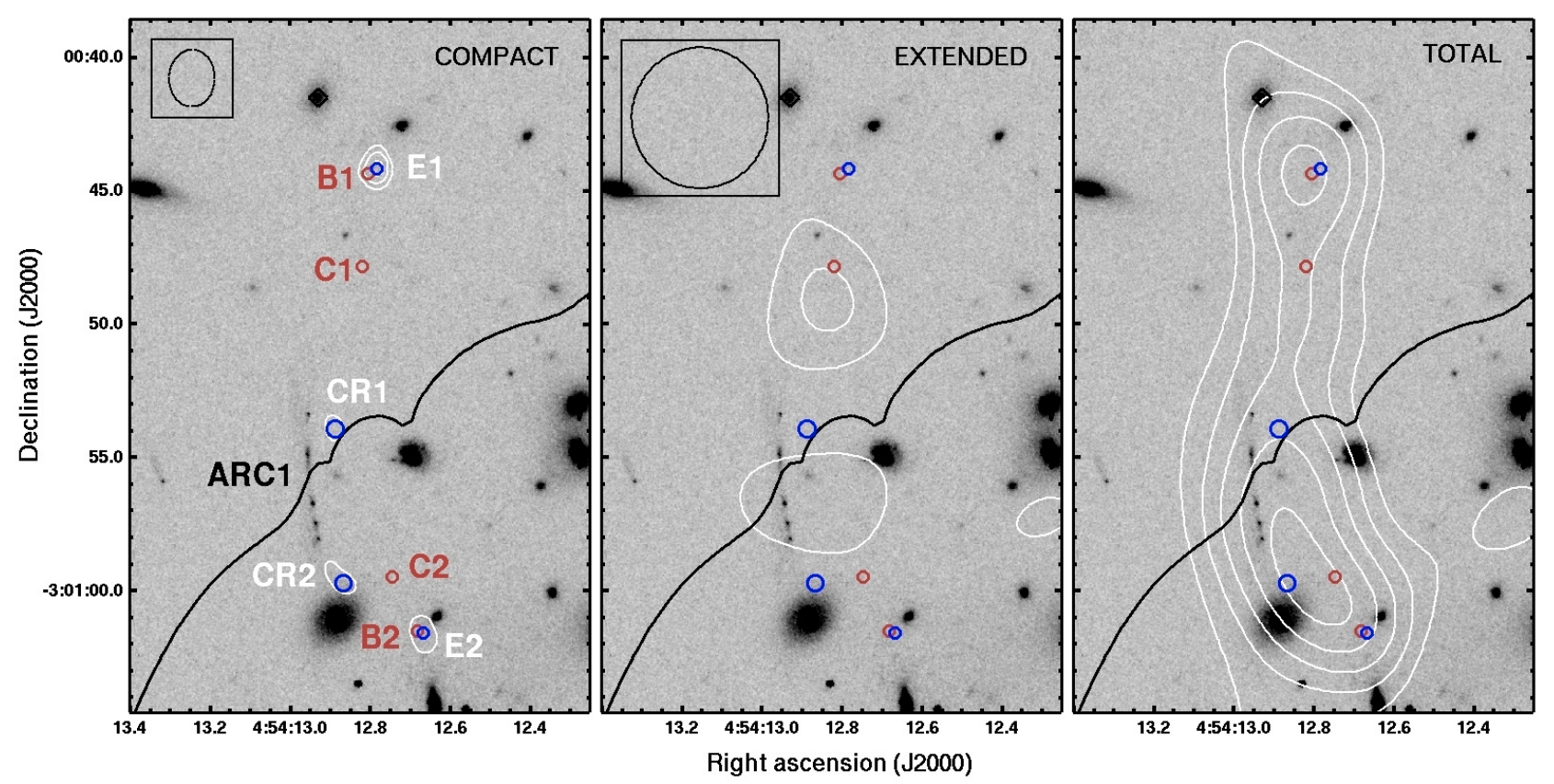

Fig. 8. Detail of the HST image in the arc region, including the critical curve at $z=2.911$ predicted by the lens model described in Sect. 5 (black line). The red circles indicate the positions of the multiply imaged EROs mentioned in Borys et al. (2004), whereas the blue circles correspond to the compact radio detections indentified in this region. The radius of the circles indicate the estimated positional errors: $\sim 0.20^{\prime \prime}$ for the EROs (astrometric error for the standard stars quoted in Takata et al. 2003), between $0.1^{\prime \prime}-0.2^{\prime \prime}$ for the radio sources (see Table 1) and $\sim 0.08^{\prime \prime}$ (half a pixel) for the optical sources. The contours correspond to the $\sim 2^{\prime \prime}$ resolution radio map presented in Fig. 4 (left panel), and two of the $\sim 5^{\prime \prime}$ resolution radio maps presented Fig. 7 (central and right panel).

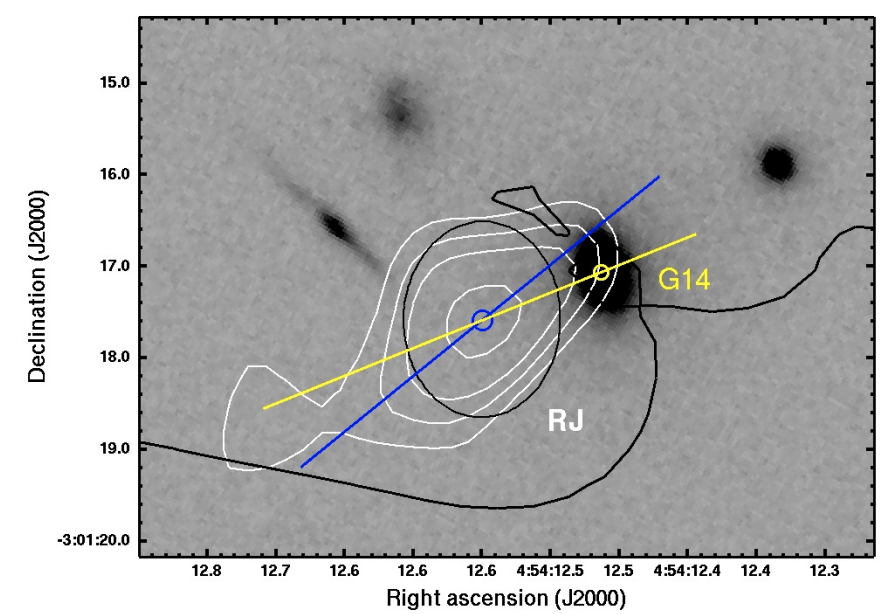

Fig. 9. Detail of the HST image in the region of RJ, including the critical curve at $z=2.911$ predicted by the lens model describe in Sect. 4.1 (black line). The contours correspond to the $\sim 2^{\prime \prime}$ resolution radio map presented in Fig. 4. The radius of the circles show the estimated positional uncertainties: $0.1^{\prime \prime}$ for RJ (see Table 1 ) and $\sim 0.08^{\prime \prime}$ (half a pixel) for G14. The blue line indicates the approximate orientation of the major axis of the radio source. For comparison, the yellow line shows the orientation of the RJ centroid respect to the galaxy G14. The black ellipse indicates the beam size of the radio map $\left(2.14^{\prime \prime} \times 1.71^{\prime \prime}\right.$ at a position angle of -1.43 ).

be linked to massive star formation ${ }^{8}$, which means that both the radio and sub-mm emission are originated in (approximately) the

\footnotetext{
${ }^{8}$ While young massive stars produce UV radiation that is re-emitted in the FIR by the surrounding dust, old massive stars die as SN producing electrons that are accelerated by the galactic magnetic field generating synchrotron radio emission.
}

same regions of the galaxy. However, the $S_{850 \mu \mathrm{m}} / S_{1.4 \mathrm{GHz}}$ flux ratio observed in sub-mm galaxies displays a broad scatter which strongly depends on the characteristic dust temperature and the redshift (e.g. Blain et al. 2002; Chapman et al. 2005). Therefore, if the observed sub-mm emission is being produced by several blended SMGs, we might find "morphological inconsistencies" like the one present in the left panel of Fig. 10 (note that the brightest peak of the radio emission is located in the region of $\mathrm{RJ}$ instead of been coincident with the brightest sub-mm peak).

Interestingly, if the $\mathrm{CC}$ model of $\mathrm{RJ}$ is subtracted from the data, the morphology of the radio emission becomes remarkably similar to the sub-mm emission (right panel of Fig. 10). This result strongly suggests that the source/sources responsible for the radio emission observed in this panel are also responsible for the bulk of the sub-mm emission. The other conclusion derived from this comparison is that, if RJ is contributing to the sub-mm emission, either its properties (redshift and/or dust temperature) are different from the properties of the source/sources associated with the other radio detections, or it has a "radio excess" due to an AGN.

Using $350 \mu \mathrm{m}, 850 \mu \mathrm{m}$ and $1.4 \mathrm{GHz}$ observations of 15 bright SMGs with spectroscopic redshifts, Kovács et al. (2006) (K06 hereafter) conclude that the FIR-radio correlation remains valid for SMGs with $z \sim 1-3$ and luminosities between $10^{11}-10^{13} \mathrm{M}_{\odot}($ except when they are radio-loud AGN). To see how our system compares with this sample, we have made a more quantitative analysis of the FIR-radio correlation using the $q_{\mathrm{L}}$ parameter introduced in K06:

$$
q_{\mathrm{L}}=\log \left(\frac{L_{\mathrm{FIR}}}{[4.52 \mathrm{THz}] L_{1.4 \mathrm{GHz}}}\right)
$$




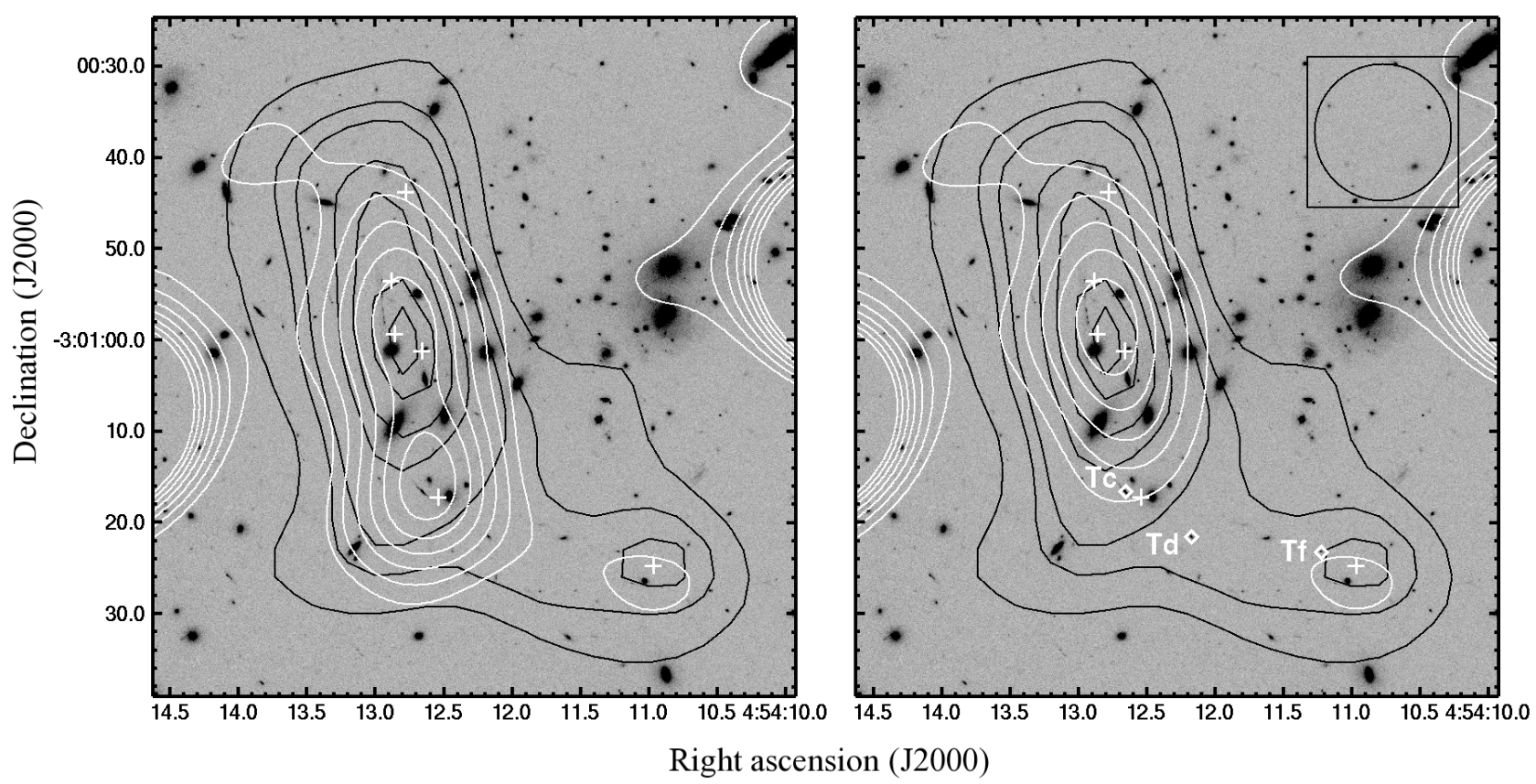

Fig. 10. Detail of the HST image of the cluster center, with the sub-mm $850 \mu \mathrm{m}$ contour map superimposed in black. The white lines correspond to the VLA 1.4 GHz contour map obtained after tapering the A+B-array data to match the resolution in sub-mm. Radio contours are drawn at 2, 3, 4, 5,6 and 7 times the $1 \sigma$ noise level of $33.93 \mu \mathrm{Jy}_{\text {beam }}{ }^{-1}$. Sub-mm contours are drawn at 4, 6, 7, 9, 10, 11 and $11.5 \mathrm{mJy}^{\prime \prime}$ beam ${ }^{-1}$. The white crosses indicate the position of the radio sources identified within the sub-mm emission region. The beam size $\left(15^{\prime \prime} \times 15^{\prime \prime}\right)$ is indicated in the top right corner. Left panel: map produced including all radio detections. Right panel: map produced after subtracting the CC model of RJ from the data. Indicated with diamonds are the positions of 3 EROs reported in Takata et al. (2003) that could be contributing to the observed sub-mm emission.

were total FIR luminosity is inferred from the flux density at $850 \mu \mathrm{m}$ assuming a grey body model:

$L_{\mathrm{FIR}}=4 \pi D_{\mathrm{L}}^{2} \Gamma(4+\beta) \zeta(4+\beta)\left(\frac{k T_{\mathrm{d}}}{h v}\right)^{4+\beta}\left(\mathrm{e}^{h v / k T_{\mathrm{d}}}-1\right) v S_{850 \mu \mathrm{m}}$

and the radio luminosity can be derived as:

$L_{1.4 \mathrm{GHz}}=4 \pi D_{\mathrm{L}}^{2} S_{1.4 \mathrm{GHz}}(1+z)^{\alpha-1}$.

In these formulas, $D_{\mathrm{L}}$ is the luminosity distance, $\beta$ is the effective emissivity index (we assume $\beta=1.5$ ), $T_{\mathrm{d}}$ is the characteristic dust temperature, $v$ corresponds to the observed sub-mm frequency, and $\alpha$ is the radio spectral index (we assume $\alpha=0.7$ ).

At the position of the sub-mm peak, the radio and sub-mm fluxes were derived using the area delimited by the $3 \sigma$ radio contour shown in the left panel of Fig. $10\left(S_{850 \mu \mathrm{m}}=15.5 \pm 2.8 \mathrm{mJy}\right.$ and $S_{1.4 \mathrm{GHz}}=241 \pm 42 \mu \mathrm{Jy}$ ). The different values of $q_{\mathrm{L}}$ derived from these fluxes as function of redshift and $T_{\mathrm{d}}$ are shown in Fig. 11. The horizontal dashed lines indicate the minimum and maximum value of $q_{\mathrm{L}}$ found in the K06 sample (discarding AGN hosts). Note that there is a wide range of $z$ and $T_{\mathrm{d}}$ for which the observed fluxes are consistent with K06. If we now assume that the radio and sub-mm emission are produced by a (lensed) star forming galaxy at $z=2.9$, only temperatures between $\sim 20-40 \mathrm{~K}$ would be allowed, which is the temperature range in which most of the K06 sources lie.

Since AGNs do not follow the FIR-radio correlation, an estimate of the $q_{\mathrm{L}}$ value for RJ could provide extra evidence to confirm/discard the possible AGN nature of this source. However, that would require a higher resolution $850 \mu \mathrm{m}$ map, to confirm the connection of RJ with a discrete sub-mm source and get an accurate estimate of its sub-mm flux. In addition, the redshift of RJ needs to be determined in order to break the degeneracy between $z$ and $T_{\mathrm{d}}$ illustrated in Fig. 11.

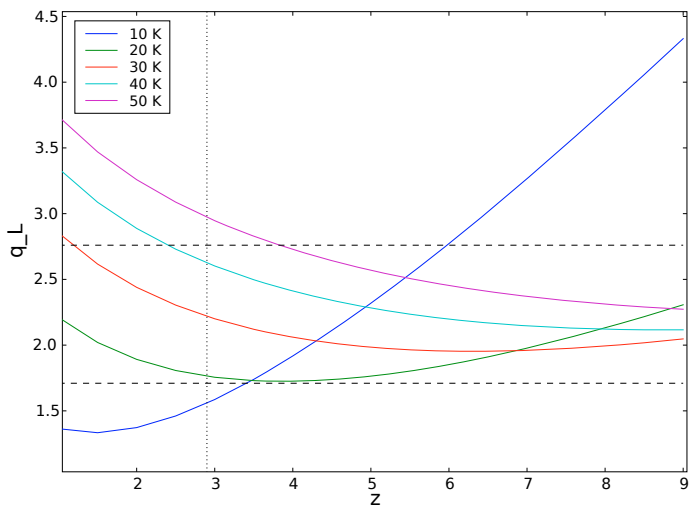

Fig. 11. Change of the parameter $q_{\mathrm{L}}$ with redshift for different characteristic dust temperatures. The horizontal dashed lines indicate the minimum and maximum value of $q_{\mathrm{L}}$ found in the SMG sample studied by Kovács et al. (2006). The dotted vertical line indicates the position of $z=2.9$.

Finally, is important to mention that, although the energy output of the majority of SMGs is dominated by star formation, about 30-50\% host an AGN (Alexander et al. 2005). Therefore, even if the source associated with RJ is an AGN, it could still be contributing to the sub-mm emission observed in that region. Other potential contributors to the observed sub-mm emission are indicated in the right panel of Fig. 10.

\section{Gravitational lens modeling of MS0451}

In order to investigate the possible lensed nature of the radio detections, we used the publicly available LENSTOOL ${ }^{9}$ code to

\footnotetext{
${ }^{9}$ http://www. oamp.fr/cosmology/lenstool/
} 


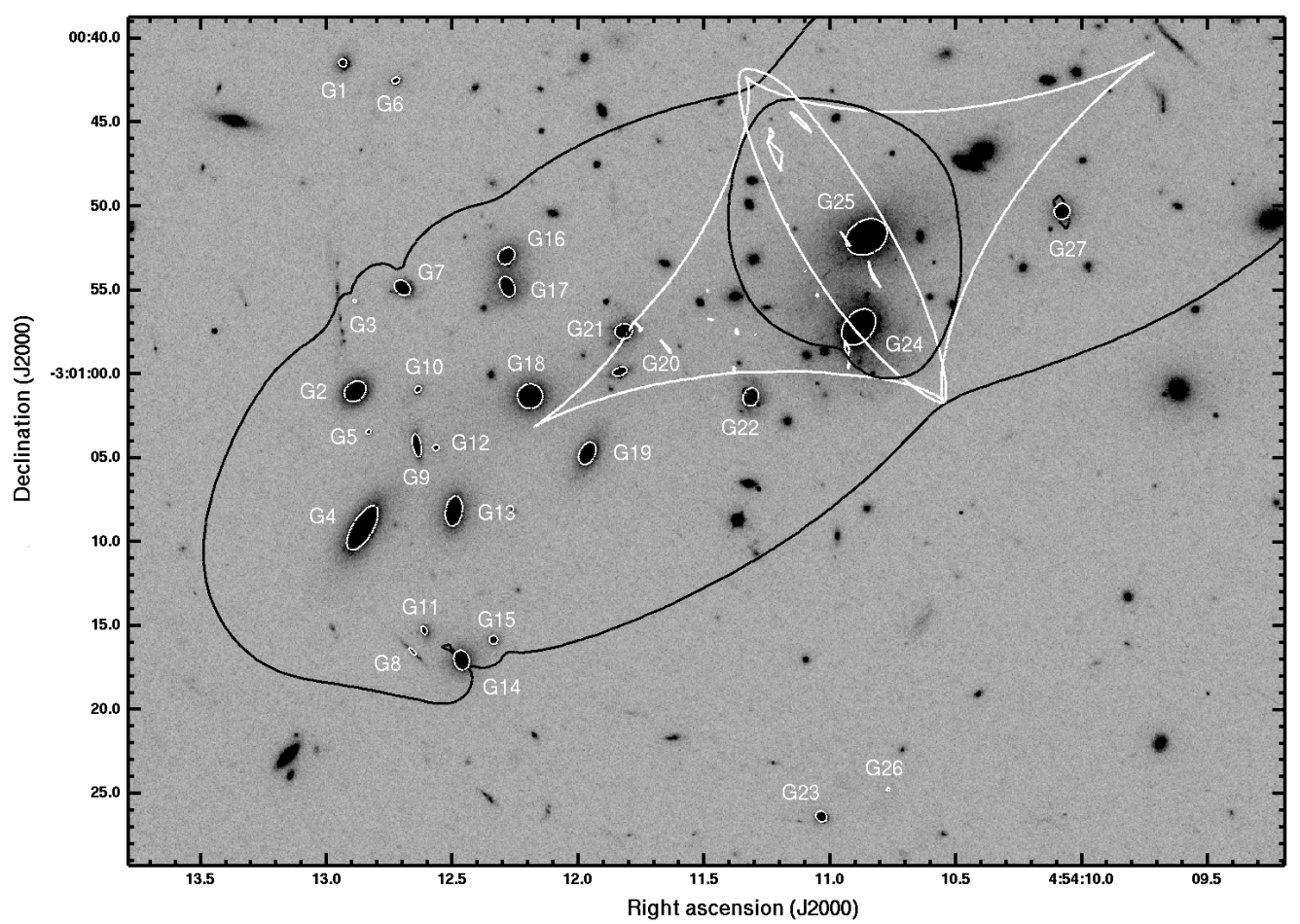

Fig. 12. Detail of the HST image of the cluster core, indicating all the galaxies that have been included in the lens model. The sizes of the ellipses correspond to the morphological parameters listed in Table 5 . The lines represent the critical curves (black) and caustics (white) predicted by the lens model at $z=2.911$.

model the mass distribution of the cluster. This modeling involves an optimization procedure, aimed to find the mass model parameter values that best reproduce the observational constrains (positions of the multiply imaged systems). For a detailed description of the strong lensing methodology followed in the LENSTOOL software we refer to Limousin et al. (2007), Jullo et al. (2007) and the appendix A2 of Smith et al. (2005).

The current best lens model of the cluster MS0451, published in B04 (B04 model hereafter), was produced with the first version of LENSTOOL (Kneib et al. 1993). This version is based on a downhill $\chi^{2}$ minimization (which can be very sensitive to local minimum in the likelihood distribution), and does not provide estimates of the errors on the optimized parameters. The latest LENSTOOL version (Jullo et al. 2007) used in this paper includes a Bayesian Monte Carlo Markov chain optimization routine, which allows to determine errors in the optimized parameters and lowers the probability of ending in local $\chi^{2}$ minimum.

\subsection{Mass model}

The cluster mass model is constructed using two different components: (i) a cluster halo, which represents the DM component on cluster scales and the baryonic intracluster gas, and (ii) 27 galaxy halos that account for individual galaxies. Both components are described using a PIEMD profile (e.g. Limousin et al. 2005; Elíasdóttir et al. 2007), parametrized by position (RA, DEC), position angle $(\theta)$, ellipticity $(\epsilon)$, core radius $\left(R_{\text {core }}\right)$, scale radius $\left(R_{\text {cut }}\right)$ and velocity dispersion $\left(\sigma_{0}\right)$. The density distribution for this profile is given by:

$\rho(r)=\frac{\rho_{0}}{\left(1+r^{2} / R_{\text {core }}^{2}\right)\left(1+r^{2} / R_{\text {cut }}^{2}\right)}$.

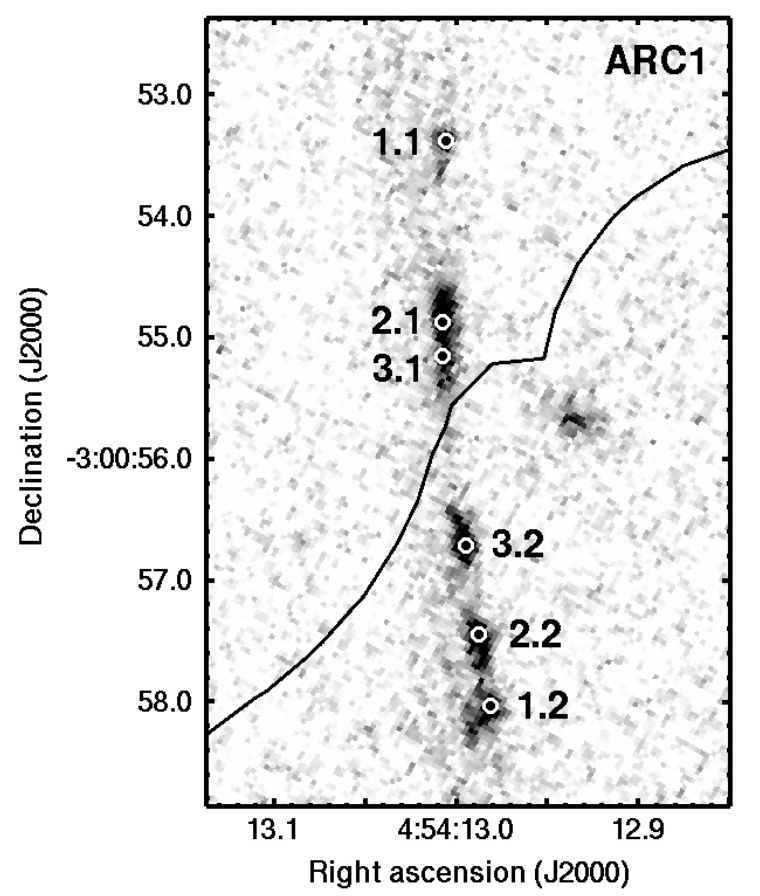

Fig. 13. Detail of the optical arc as seeing in the HST image. The circles indicate the positions of the three sets of mirror images listed in Table 6.

The galaxy-scale component contains the cluster members located relatively close to the area were the multiple images are formed, because they are the ones that have the strongest effect in the potential of that region. We also included some fainter galaxies located close to the strong lensing constraints, since they are known to perturb the strong lensing configuration 
Table 4. Conunterparts of the radio detections.

\begin{tabular}{lcccc}
\hline \hline $\begin{array}{l}\text { Radio } \\
\text { source }\end{array}$ & $\begin{array}{c}\text { Optical/IR } \\
\text { source }\end{array}$ & $\begin{array}{c}d_{\text {obs }} \\
(\operatorname{arcsecs})\end{array}$ & $\begin{array}{c}r_{\text {src }} \\
(\operatorname{arcsecs})\end{array}$ & $\begin{array}{c}P_{\text {assoc. }} \\
(\%)\end{array}$ \\
\hline E1 & B1 ${ }^{*}$ & 0.38 & 0.6 & 67.8 \\
\hline CR1 & ARC 1.1 & 1.12 & 0.15 & $<1$ \\
& ARC 2.1 & 1.40 & 0.25 & $<1$ \\
& ARC 3.1 & 1.60 & 0.20 & $<1$ \\
\hline CR2 & G2 & 1.39 & 0.70 & $<1$ \\
& C2 ${ }^{*}$ & 1.82 & 0.60 & $<1$ \\
& ARC 1.2 & 1.92 & 0.15 & $<1$ \\
\hline E2 & B2 ${ }^{*}$ & 0.26 & 0.60 & 79.29 \\
& G10 & 0.74 & 0.23 & $<1$ \\
\hline E3 & $4.3^{*}$ & 0.77 & 0.60 & 20.7 \\
& $5.3^{*}$ & 1.22 & 0.30 & $<1$ \\
& G23 & 1.54 & 0.35 & $<1$ \\
\hline RJ & G8 & 1.91 & 0.30 & $<1$ \\
& G11 & 2.40 & 0.26 & $<1$ \\
& G14 & 1.39 & 0.60 & $<1$ \\
\hline
\end{tabular}

The columns show: possible counterparts of the radio detections (Optical/IR source), mesured offset between the radio source and the optical/IR source $\left(d_{\mathrm{obs}}\right)$, radius of the optical/IR source $\left(r_{\text {src }}\right)$ and probability that the centroid of the radio source lies inside the area of the optical/IR source $\left(P_{\text {assoc. }}\right)$. The coordinates of the counterpart sources can be found in Tables 5 and 6 . Sources which positions were taken from the $K^{\prime}$ band image are indicated with an asterisk (otherwise we used the HST coordinates). The positions of the radio sources used to calculate $d_{\text {obs }}$ correspond to the values derived with MAXFIT in the aligned radio map.

(Meneghetti et al. 2007). The details of our galaxy catalog are listed in Table 5, and their positions and sizes are displayed in Fig. 12. Note that the galaxy catalog used in the B04 model (39 galaxies in total) includes cluster members located farther away, but not the faint galaxies mentioned before.

\subsection{Multiple images}

Figure 13 shows a close up of ARC1 as seeing on the ACS image. From its knotted structure we can identify 3 sets of mirror images, each of which should have its own counter-image. However, since it is not possible to distinguish the different knots in the de-magnified image of the arc (ARC1 ci, see Fig. 14), we assigned the same position to the counter-image of each set of constraints coming from the arc.

The positions for the EROs used in our model come from the Subaru $K^{\prime}$ band image published in Takata et al. (2003), which is deeper and has higher resolution than the CFHT $K^{\prime}$ band image published by B04. Based on their model, B04 identified the NIR source labeled $\mathrm{B} 3 / \mathrm{C} 3$ as the result of the blending between the counter-images of B and C. However, we have detected a faint source (labeled "5.3" in Fig. 14) located very close to B3/C3 in the Subaru $K^{\prime}$ band image. This source was not reported in Takata et al. (2003) because it is too faint to be classified as a DRG ${ }^{10}$ (Tadafumi Takata, private communication). Since it is possible that this source is the resolved counter-image of ERO C, we decided to include it as a constraint in the new model. The full set of constraints used for the optimization is listed in Table 6.

${ }_{10}$ Distant Red Galaxies, photometrically defined as $J-K \mathrm{~s}>2.3$.
Table 5. Galaxy catalog for the new lens model of MS0451.

\begin{tabular}{lcccccc}
\hline \hline ID & $\begin{array}{c}\text { RA }\left(+4^{\mathrm{h}}\right. \\
\text { 52000 }(\mathrm{s})\end{array}$ & $\begin{array}{c}\text { Dec }\left(-3^{\circ}\right) \\
\text { J2000 }\left({ }^{\prime},{ }^{\prime \prime}\right)\end{array}$ & $\begin{array}{c}\mathrm{a} \\
\text { (arcsec) }\end{array}$ & $\begin{array}{c}\mathrm{b} \\
(\operatorname{arcsec})\end{array}$ & $\begin{array}{c}\theta \\
(\mathrm{deg})\end{array}$ & $\begin{array}{c}\mathrm{mag} \\
(F 814 W)\end{array}$ \\
\hline $01^{*}$ & 12.933 & $00: 41.494$ & 0.24 & 0.23 & 65 & 21.9511 \\
02 & 12.884 & $01: 01.056$ & 0.70 & 0.55 & 40 & 20.1111 \\
$03^{*}$ & 12.886 & $00: 55.685$ & 0.12 & 0.09 & -28 & 24.6286 \\
04 & 12.855 & $01: 09.205$ & 1.50 & 0.60 & 59 & 19.0257 \\
$05^{*}$ & 12.831 & $01: 03.476$ & 0.17 & 0.15 & 55 & 23.2229 \\
$06^{*}$ & 12.723 & $00: 42.529$ & 0.24 & 0.17 & 30 & 22.3768 \\
07 & 12.697 & $00: 54.889$ & 0.53 & 0.40 & 140 & 20.8427 \\
$08^{*}$ & 12.655 & $01: 16.586$ & 0.30 & 0.10 & -44 & 22.5806 \\
$09^{*}$ & 12.639 & $01: 04.288$ & 0.70 & 0.25 & -80 & 20.7576 \\
$10^{*}$ & 12.634 & $01: 00.949$ & 0.23 & 0.17 & 50 & 22.4744 \\
$11^{*}$ & 12.610 & $01: 15.312$ & 0.26 & 0.16 & -69 & 22.1831 \\
$12^{*}$ & 12.563 & $01: 04.426$ & 0.19 & 0.19 & 20 & 22.5221 \\
13 & 12.492 & $01: 08.202$ & 0.90 & 0.50 & 81 & 19.6345 \\
14 & 12.461 & $01: 17.075$ & 0.60 & 0.45 & 105 & 20.0909 \\
$15^{*}$ & 12.334 & $01: 15.892$ & 0.25 & 0.25 & -15 & 21.9945 \\
16 & 12.283 & $00: 53.001$ & 0.55 & 0.45 & 50 & 20.3429 \\
17 & 12.280 & $00: 54.816$ & 0.64 & 0.42 & 115 & 20.0471 \\
18 & 12.189 & $01: 01.348$ & 0.75 & 0.73 & 10 & 19.5202 \\
19 & 11.962 & $01: 04.756$ & 0.70 & 0.46 & 63 & 20.1070 \\
$20^{*}$ & 11.833 & $00: 59.879$ & 0.45 & 0.25 & 16 & 21.1736 \\
21 & 11.817 & $00: 57.484$ & 0.53 & 0.45 & 15 & 20.3933 \\
22 & 11.312 & $01: 01.400$ & 0.55 & 0.45 & 66 & 20.6069 \\
$23^{*}$ & 11.032 & $01: 26.400$ & 0.35 & 0.30 & -40 & 21.4960 \\
24 & 10.884 & $00: 57.231$ & 1.20 & 0.85 & 50 & 18.1360 \\
25 & 10.853 & $00: 51.865$ & 1.30 & 1.00 & 30 & 18.1454 \\
$26^{*}$ & 10.767 & $01: 24.772$ & 0.12 & 0.08 & 21 & 25.2539 \\
27 & 10.076 & $00: 50.338$ & 0.47 & 0.47 & 56 & 20.5351 \\
\hline
\end{tabular}

The colums show: galaxy number (ID) galaxy coordinates (RA, Dec), semi-major axes (a), semi-minor axes (b), position angle $(\theta)$ and magnitude in the HST image (mag). An asterisk in the ID number indicate galaxies that were not included in the model presented in Borys et al. (2004).

\subsection{Optimization}

The set of free parameters used during the optimization are: (i) all the parameters that characterize the cluster halo (except $\left.R_{\text {core }}\right)$ and (ii) the velocity dispersion $\left(\sigma_{0}^{*}\right)$ and scale radius $\left(R_{\text {cut }}^{*}\right)$ of a galaxy at $z=z_{\text {cluster }}$ with a typical luminosity $L^{*}$ that corresponds to an observed magnitude of $m=18.8$.

The $\sigma_{0}$ and $R_{\text {cut }}$ of all the galaxy halos included in the mass model are derived from the luminosity of their associated galaxy using the following empirical scaling relations:

$R_{\text {cut }}=R_{\text {cut }}^{*}\left(\frac{L}{L^{*}}\right)^{1 / 2} \quad \sigma_{0}=\sigma_{0}^{*}\left(\frac{L}{L^{*}}\right)^{1 / 4}$

The scaling relation for $\sigma_{0}$ assumes that mass traces light, and its origin resides in the Tully-Fisher and Faber-Jackson relations. The scaling relation for the radial parameter assumes that the mass-to-light ratio is constant for all galaxies. The zero point of these relations is set by the magnitude of the $L^{*}$ galaxy. The rest of the parameters of all galaxy halos (RA, Dec, $\theta$ and $\epsilon$ ) are fixed to the values measured from the light distribution of the related galaxy, while their $R_{\text {core }}$ is set to zero.

The ranges in which the free parameters are allowed to vary during the optimization are listed in Table 7 . Note that the scale radius describes the properties of the mass distribution on scales much larger than the radius over which the multiple images can be found. Therefore, since strong lensing cannot give any reliable constrains on this parameter, its value is set to an arbitrary large number $(1500 \mathrm{kpc})$. The ranges adopted for the 
Table 6. Constraints for the new lens model of MS0451.

\begin{tabular}{ccccc}
\hline \hline Source name & ID & $\begin{array}{c}\text { RA }\left(+4^{\mathrm{h}} 54^{\mathrm{m}}\right) \\
\mathrm{J} 2000(\mathrm{~s})\end{array}$ & $\begin{array}{l}\text { Dec }\left(-3^{\circ}\right) \\
\mathrm{J} 2000\left({ }^{\prime},{ }^{\prime \prime}\right)\end{array}$ & Band \\
\hline & $1.1^{*}$ & 12.956 & $0: 53.379$ & $F 814 W$ \\
ARC1 & $1.2^{*}$ & 12.931 & $0: 58.029$ & \\
& $1.3^{*}$ & 11.124 & $1: 26.641$ & \\
\hline & 2.1 & 12.958 & $0: 54.869$ & $F 814 W$ \\
ARC1 & 2.2 & 12.938 & $0: 57.446$ & \\
& 2.3 & 11.124 & $1: 26.641$ & \\
\hline & 3.1 & 12.958 & $0: 55.147$ & $F 814 W$ \\
ARC1 & 3.2 & 12.945 & $0: 56.710$ & \\
& 3.3 & 11.124 & $1: 26.641$ & \\
\hline B1 & $4.1^{*}$ & 12.806 & $0: 44.344$ & $K^{\prime}$ band \\
B2 & $4.2^{*}$ & 12.684 & $1: 01.488$ & \\
B3 & $4.3^{*}$ & 10.927 & $1: 24.795$ & \\
\hline C1 & 5.1 & 12.822 & $0: 47.834$ & $K^{\prime}$ band \\
C2 & 5.2 & 12.747 & $0: 59.481$ & \\
C3 & 5.3 & 10.897 & $1: 25.627$ & \\
\hline RC1 & 6.1 & 12.890 & $0: 53.907$ & $1.4 \mathrm{GHz}$ \\
RC2 & 6.2 & 12.868 & $0: 59.696$ & \\
\hline
\end{tabular}

Sources used as constraints in the lens model presented by Borys et al. (2004) are indicated with an asterisk in their ID number.

Table 7. Ranges in which the free parameters are allowed to vary during the lens model optimization.

\begin{tabular}{cc}
\hline \hline Parameter & Opt. range \\
\hline $\mathrm{X}$ & $\pm 6^{\prime \prime}$ from BCG center \\
$\mathrm{Y}$ & $\pm 6^{\prime \prime}$ from BCG center \\
$\epsilon$ & $0-0.7$ \\
$\theta$ & $0-180 \mathrm{deg}$ \\
$\sigma_{0}$ & $900-1800 \mathrm{~km} \mathrm{~s}^{-1}$ \\
$R_{\text {core }}$ & $30-120 \mathrm{kpc}^{-1}$ \\
\hline$\sigma_{0}^{*}$ & $120-140 \mathrm{~km} \mathrm{~s}^{-1}$ \\
$R_{\text {cut }}^{*}$ & $30-180 \mathrm{kpc}$ \\
\hline
\end{tabular}

$L^{*}$ galaxy are motivated by galaxy-galaxy lensing studies in clusters (Natarajan et al. 1998, 2002a,b; Limousin et al. 2007).

Since the redshift of the EROs has not been confirmed spectroscopically, the modeling procedure was carried out in four steps. In the first step, only the sets of images from ARC1 at $z_{\text {spect }}=2.911$ where used as constraints in the optimization. The resultant best model was then re-optimized, including the constraints provided by ERO B but leaving $z_{\mathrm{B}}$ as free parameter. As a result, the predicted redshift of ERO $\mathrm{B}$ is $z_{\mathrm{B}}=2.93 \pm 0.13$. In the third step, the constraints from ERO $\mathrm{C}$ were used in a new re-optimization of the best model obtained in the previous step, assuming $z_{\mathrm{B}}=2.9$ and leaving $z_{\mathrm{C}}$ as free parameter. This provides a $z_{\mathrm{C}}=2.85 \pm 0.06$, which is the same redshift that $\mathrm{B} 04$ derived for the ERO pair. In the final step, the previous best model is re-optimized (in the image plane) including all the constraints with $z=2.911$.

The Bayesian MCMC optimization routine included in the new version of LENSTOOL provides two kinds of outputs: (i) the likelihood of reproducing the observed constraints, independently derived for each free parameter of the mass model, and (ii) the set of model parameters that provides the best fit to the input data. The most likely values for the model parameters obtained from these histograms are listed in Table 8, whereas the

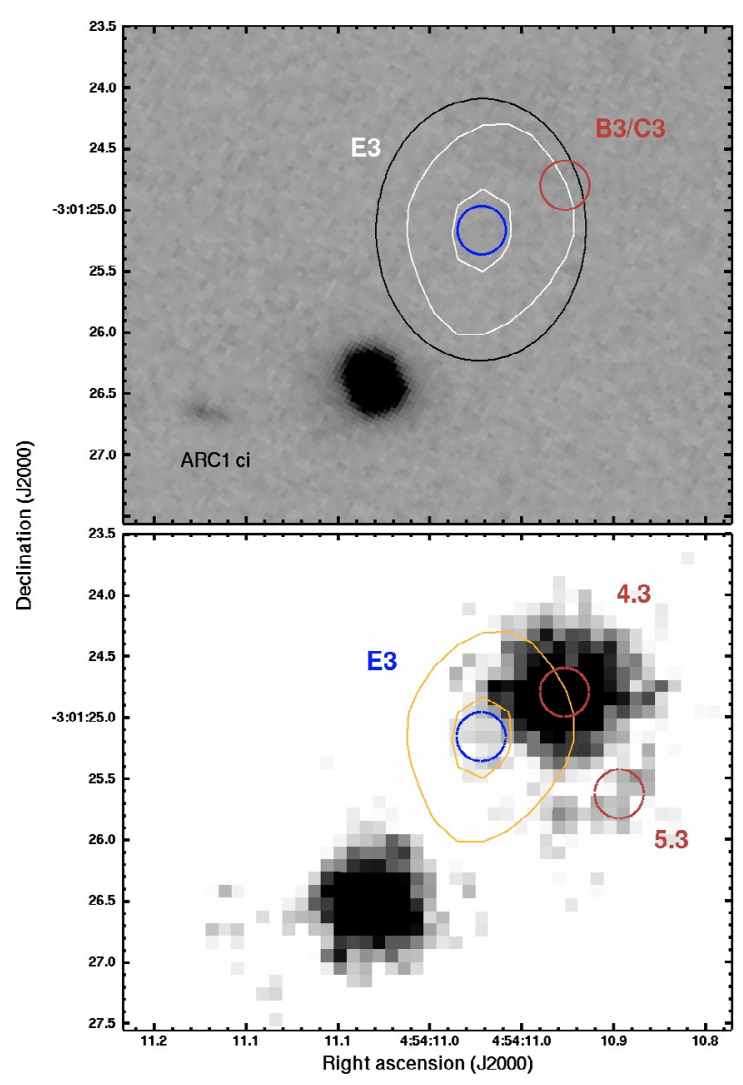

Fig. 14. Detail of the source E3, including its radio contours as presented in Fig. 4. Top panel: HST image of the region, including the positions of the counter images of the optical arc (ARC1 ci) and the ERO pair (B3/C3) reported by Borys et al. (2004). Bottom panel: $K^{\prime}$ band image of the region, indicating the constraints used in the lens model (4.3 and 5.3). The radius of the circles show the estimated positional uncertainties: $\sim 0.20^{\prime \prime}$ for the $K^{\prime}$ band sources (astrometric error for the standard stars quoted in Takata et al. 2003), 0.2" for E3 (see Table 1) and $\sim 0.08^{\prime \prime}$ (half a pixel) for the optical sources. The black ellipse indicates the beam size of the radio map $\left(2.14^{\prime \prime} \times 1.71^{\prime \prime}\right.$ at a position angle of -1.43$)$.

parameters of the best model (MFINAL hereafter) are listed in Table 9.

The image positions are well reproduced, with image plane positional $\mathrm{rms}$ differences between $\sim 0.1^{\prime \prime}$ and $0.3^{\prime \prime}$ (see Table 10). The projected mass within the Einstein radius (here approximated by the ARC1 distance from the center, is $M_{2 \mathrm{D}}\left(30^{\prime \prime}\right)=1.73 \times 10^{1} 4 M_{\odot}$.

\subsection{Analysis of the radio data using the new lens model}

Since CR1 and CR2 are located in a region with several multiply-imaged systems (see Fig. 8), it is conceivable that they might also constitute a set of mirror images. To test this hypothesis, we redo the optimization using the positions of these two radio sources as an additional set of constraints (system 6 , see Table 6), first assuming $z_{\mathrm{CR}}=2.911$ and then considering $z_{\mathrm{CR}}$ as a free parameter. A summary of the properties of the resultant best models (MZFIX and MZFREE hereafter) is presented in Tables 10 and 11.

The optimization with $z$ free results in a predicted redshift for system 6 of $z_{\mathrm{CR}}=2.4 \pm 0.074$, which is not consistent with $z=2.911$. If we compare the probability of MZFIX and MZFREE to reproduce the observations (the evidence, see Table 11), it is 
Table 8. Most likely mass model parameters.

\begin{tabular}{cccccccc}
\hline \hline Clump & $\begin{array}{c}\mathrm{X} \\
\left({ }^{\prime \prime}\right)\end{array}$ & $\begin{array}{c}\mathrm{Y} \\
\left({ }^{\prime \prime}\right)\end{array}$ & $\epsilon$ & $\begin{array}{c}\theta \\
(\mathrm{deg})\end{array}$ & $\sigma_{0}$ & $\begin{array}{c}r_{\text {core }} \\
(\mathrm{kpc})\end{array}$ & $\begin{array}{c}r_{\text {cut }} \\
(\mathrm{kpc})\end{array}$ \\
\hline Cluster & $-0.5_{-1.2}^{+4.1}$ & $2.7 \pm 1.5$ & $0.69_{-0.1}^{+0.0}$ & $31.3 \pm 1.1$ & $1144.8 \pm 36$ & $60.8 \pm 24$ & {$[1500]$} \\
$\mathrm{L}^{*}$ elliptical galaxy & - & - & - & - & $135.1 \pm 43.9$ & {$[0]$} & $30.1_{-4.9}^{+43.6}$ \\
\hline
\end{tabular}

The columns show: position respect to the brightest cluster galaxy (X,Y), ellipticity of the mass distribution $\left(\epsilon\right.$, expressed as $a^{2}-b^{2} / a^{2}+b^{2}$, were $a$ and $b$ are the semi-major and semi minor axes of the ellipse that describes the light distribution of the clump), position angle $(\theta)$, velocity dispersion $\left(\sigma_{0}\right)$, core radius $\left(r_{\text {core }}\right)$ and scale radius $\left(r_{\text {cut }}\right)$. Error bars correspond to $1 \sigma$ confidence level as inferred from the MCMC optimization. Values into brackets are not optimized. When the posterior distribution is not Gaussian, we report the mode and asymmetric error bars. The coordinates of the brightest cluster galaxy are $\mathrm{RA}=4^{\mathrm{h}} 54^{\mathrm{m}} 10.87^{\mathrm{s}}$, Dec $=-3^{\circ} 0^{\prime} 54.00^{\prime \prime}$.

Table 9. Best mass model.

\begin{tabular}{cccccccc}
\hline \hline Clump & $\begin{array}{c}\mathrm{X} \\
\left({ }^{\prime \prime}\right)\end{array}$ & $\begin{array}{c}\mathrm{Y} \\
\left({ }^{\prime \prime}\right)\end{array}$ & $\epsilon$ & $\begin{array}{c}\sigma_{0} \\
(\mathrm{deg})\end{array}$ & $\begin{array}{c}r_{\text {core }} \\
\left(\mathrm{km} \mathrm{s}^{-1}\right)\end{array}$ & $\begin{array}{c}r_{\text {cut }} \\
(\mathrm{kpc})\end{array}$ & \begin{tabular}{c}
$\mathrm{kpc})$ \\
\hline Cluster
\end{tabular} \\
-0.771414 & 2.256631 & 0.695267 & 31.146240 & 1117.322994 & 7.667450 & {$[1500]$} \\
$L^{*}$ elliptical galaxy & - & - & - & - & 150.421411 & {$[0]$} & 3.880257 \\
\hline
\end{tabular}

The columns show: position respect to the brightest cluster galaxy (X,Y), ellipticity of the mass distribution $\left(\epsilon\right.$, expressed as $a^{2}-b^{2} / a^{2}+b^{2}$, were $a$ and $b$ are the semi-major and semi minor axes of the ellipse that describes the light distribution of the clump), position angle $(\theta)$, velocity dispersion $\left(\sigma_{0}\right)$, core radius $\left(r_{\text {core }}\right)$ and scale radius $\left(r_{\text {cut }}\right)$. Values into brackets are not optimized. The coordinates of the brightest cluster galaxy are $\mathrm{RA}=4^{\mathrm{h}} 54^{\mathrm{m}} 10.87^{\mathrm{s}}, \mathrm{DEC}=-3^{\circ} 0^{\prime} 54.00^{\prime \prime}$.

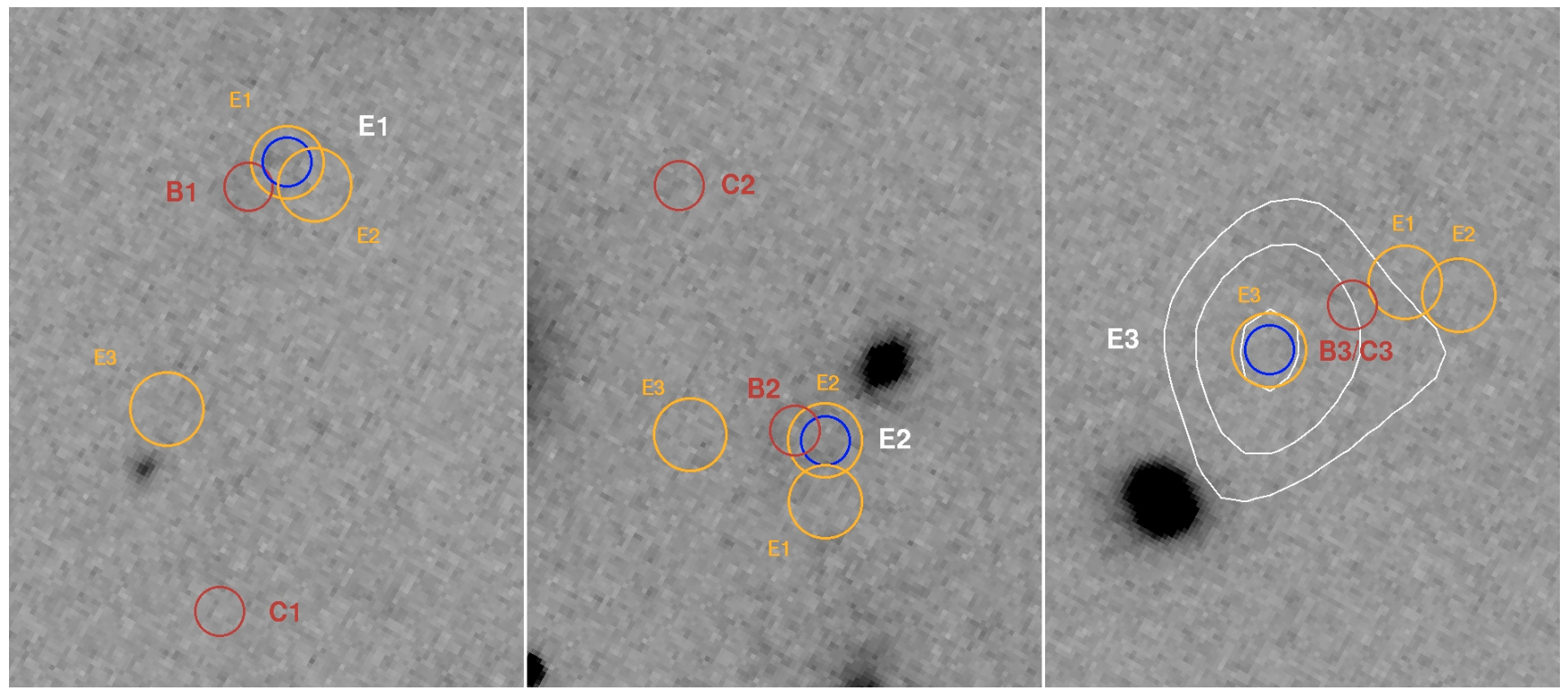

Fig. 15. Analisis of the lensing nature of E1, E2 and E3 with the lens model MFINAL. The orange circles indicate the image positions predicted after tracing each of the three radio sources independently into the source plane and lens them back into the image plane. The radius of the circles correspond to $0.3^{\prime \prime}$. The rest of the labels are the same used in Fig. 8.

clear that MZFREE is preferred over MZFIX. Note also that the rms difference between the observed and predicted image positions for system 6 is three times worse for MZFIX compared with MZFREE (see Table 10). On the other hand, the total $\chi^{2}$ of MZFREE for all the optical/IR multiply imaged systems $\left(\chi^{2}\right.$ optical, see Table 11) is just slightly worse that the corresponding $\chi^{2}$ of MFINAL, which means that MFINAL and MZFREE are equally good. Note that the evidence cannot be used to compare these two models because the number of free parameters in each of them is different.

Therefore, unless the positional uncertainty of CR1 and CR2 could be as large as $\sim 1^{\prime \prime}$ (which seems unlikely, since the positional error for these sources derived from the observations is $\left.0.2^{\prime \prime}-0.3^{\prime \prime}\right)$, these results indicate that the lens model favors an scenario in which CR1 and CR2 (if assumed to be mirror images) are produced by a radio source that is not located at the same redshift as the optical arc. However, as discussed in Sect. 3.2, CR1 and CR2 seem to be two relatively compact $\left(\sim 2^{\prime \prime}\right)$ regions of an extended $\left(\sim 5^{\prime \prime}\right)$ radio source. Therefore, it is possible that this extended radio emission is a multiply imaged structure produced by a source at $z=2.9$, in which CR1 and CR2 are not mirror images. Until the structure of this extended radio emission can be robustly mapped with deeper observations and included in the modeling process, the posibility that CR1 and CR2 are associated with a lensed source at redshift 2.9 remains open.

As mentioned in Sect. 4.1, the multiple images of ERO B have a non-negligible probability of being associated with the 
Table 10. Results of the best models for each set of constraints.

\begin{tabular}{ccccccc}
\hline \hline System & $\begin{array}{c}\chi^{2} \\
\text { MFINAL }\end{array}$ & $\begin{array}{c}\chi^{2} \\
\text { MZFIX }\end{array}$ & $\begin{array}{c}\chi^{2} \\
\text { MZFREE }\end{array}$ & $\begin{array}{c}\text { rms } \\
\text { MFINAL }\end{array}$ & $\begin{array}{c}\text { rms } \\
\text { MZFIX }\end{array}$ & $\begin{array}{c}\text { rms } \\
\text { MZFREE }\end{array}$ \\
\hline 1 & 3.89 & 6.71 & 4.04 & 0.34 & 0.45 & 0.35 \\
2 & 2.02 & 0.68 & 1.28 & 0.25 & 0.14 & 0.20 \\
3 & 1.33 & 0.74 & 0.95 & 0.20 & 0.15 & 0.17 \\
4 & 3.86 & 8.53 & 6.32 & 0.34 & 0.51 & 0.44 \\
5 & 0.50 & 1.48 & 0.62 & 0.12 & 0.21 & 0.14 \\
6 & & 35.46 & 3.70 & & 1.26 & 0.41 \\
\hline
\end{tabular}

The columns show: ID of each set of constraints (System), reduced $\chi^{2}$ obtained for each model ( $\chi^{2}$ final, zfix and zfree) and average separation between the observed and predicted position of the lensed images for each model (rms final, zfit and zfree).

Table 11. General results of the best models.

\begin{tabular}{ccccc}
\hline \hline Model & $\chi^{2}$ Optical & $\chi^{2}$ Radio & Aver rms & Log(evidence) \\
\hline MFINAL & 11.60 & - & 0.26 & -24.503 \\
MZFIX & 18.14 & 35.46 & 0.53 & -45.340 \\
MZFREE & 13.21 & 3.70 & 0.30 & -29.741 \\
\hline
\end{tabular}

The columns show: Nickname of the model (Model), sum of the reduced $\chi^{2}$ of all the optical/NIR sets of constraints $\left(\chi^{2}\right.$ optical), reduced $\chi^{2}$ for the radio set of constraints $\left(\chi^{2}\right.$ radio), average separation between the observed and predicted position of all the lensed images used as constraints (aver rms), probability of the model to reproduce the observations $(\log ($ evidence $))$.

radio detections E1, E2 and E3. However, the probability that E3 and $\mathrm{B} 3 / \mathrm{C} 3$ are associated is significantly lower than for E1-B1 and E2-B2. For this reason, we used MFINAL to check if the positions of E1, E2 and E3 would be consistent with a set of three images produced by a radio source at $z=2.9$. This was done in the following way: for each radio detection, its position was traced back into the source plane, and the resultant source was lensed again into the image plane. In this way, the model provided three predicted positions for each radio source, one of them being the position used as input.

The results (shown in Fig. 15) indicate that E3 cannot be interpreted as the radio counterpart of $\mathrm{B} 3 / \mathrm{C} 3$, since the images produced by $\mathrm{E} 1$ and $\mathrm{E} 2$ in the counter image region are located on the right side of $\mathrm{B} 3 / \mathrm{C} 3$ (unlike in the case of $\mathrm{E} 1$ and $\mathrm{E} 2$ ), at a distance which is inconsistent with the average rms of MFINAL. A possible scenario that can explain this result is that E1 and E2 are multiple images (but we don't detect the associated counterimage because it is too faint) and E3 is a non-lensed radio galaxy that it is serendipitously lying in this region. However, as it will be discussed in the next section, it is also possible to explain E3 as a lensed image produced by a source at $z=2.9$ under the merger scenario proposed in B04.

\section{Discussion: the merger scenario}

Figure 8 shows a detail of the HST image at the position of the sub-mm peak, where ARC1 and the EROs are located. The contour map of the right panel indicates the total radio emission observed in this region, whereas the left and central panels show the compact and extended component. The black line correspond to the critical curve at $z=2.9$ predicted by the lens model of the cluster described in Sect. 5.

If ARC1 and the EROs are indeed multiple images produced by three different regions of a merger at $z=2.9$, the rest of the merger lying between them should also be multiply-imaged. Hence, if the merger contains a lot of dust (as expected from a high- $z$ SMG), this images would not be seen in the optical, but they would show up in the radio.

Under this scenario, the fact that CR1 and CR2 do not seem to have optical counterparts, and lie in the region between ARC1 and the ERO images, suggests that they could be lensed images associated with the highly-obscured center of the merger. However, as discussed in Sect. 5.4, they can also be interpreted as a set of mirror images produced by another radio galaxy located at a different redshift. The real strong evidence in favor of the merger hypothesis comes from the extended component in which CR1 and CR2 seem to be embedded, which provides $38 \%$ of the observed radio flux in the region of the sub-mm peak. As is clearly shown in the central panel of Fig. 8, this extended component constitutes a bridge of emission between the compact radio sources, as expected from the multiply imaged dustobscured material of the merger located between the optical and NIR emitting regions. In complete agreement with this scenario, the shape of the total radio emission in the right panel of Fig. 8 follows the distribution of the optical arc and the ERO images remarkably well.

In this case, we can also expect the extended emission to be multiply imaged like ARC1 and the EROs, producing a counterimage in the region between $\mathrm{ARC} 1 \mathrm{ci}$ and $\mathrm{B} 3 / \mathrm{C} 3$ (see Fig. 14). Since the images in this region are de-magnified, it is likely that the expected radio counterpart of $\mathrm{B} 3 / \mathrm{C} 3$, and the counter image associated with the extended emission, are blended. That would shift the peak of the total observed radio emission towards ARC1 ci, explaining why the probability derived for E3-B3/C3 is much lower than what it is found for E1-B1 and E2-B2.

Finally, it is also not surprising that E1-B1 and E2-B2 are not identified as robust $(\geq 95 \%)$ counterpart pairs. With the cluster magnification generating a high resolution (distorted) view of the inner structure of the merger, the observed offset between E1B1 and E2-B2 could be real, indicating that the NIR and radio emissions are arising from slightly different regions inside the merger.

\section{Summary and conclusions}

SMM J04542-0301 is an elongated region of bright sub-mm emission located in the core of the cluster MS0451.6-0305. It has been suggested (Borys et al. 2004) to be partially produced by a multiply-imaged $z=2.9$ merger which contains a LBG (lensed as ARC1 and ARC1 ci), and two EROs (lensed as B1, $\mathrm{B} 2, \mathrm{C} 1, \mathrm{C} 2$ and $\mathrm{B} 3 / \mathrm{C} 3)$. Given the low resolution and poor positional accuracy of the sub-mm map, it is very difficult to confirm the connection between SMM J04542-0301 and the opti$\mathrm{cal} / \mathrm{NIR}$ lensed images. However, high resolution radio interferometric observations can help to establish this connection thanks to the FIR-radio correlation, which has being found to be valid for SMGs (Kovács et al. 2006; Vlahakis et al. 2007; Ibar et al. 2008; Michałowski et al. 2009).

In a previous paper (Berciano Alba et al. 2007), we reported on the detection of $1.4 \mathrm{GHz}$ radio emission coincident with this system using VLA archival data. Now, following a more sophisticated data reduction procedure, the previous B-array observations have being re-reduced and combined with new high resolution A-array observations. The resultant data set has been used to produced two radio maps of the cluster core:

(i) a deep $(\sim 10 \mu \mathrm{Jy})$, high resolution $\left(\sim 2^{\prime \prime}\right)$ map, to compare with the optical/NIR images. In this map we have detected 
5 sources (E1, E2, CR1, CR2 and E3) located near the optical/NIR lensed images, and one source $(\mathrm{RJ})$ located in the central region of the sub-mm emission (see Fig. 4). Despite the fact that some of the sources have $S N R<5$, the fact that the $-4 \sigma$ peaks lie far from the central target region adds confidence to the reality of the sources.

(ii) a 15" resolution map, to compare with SMM J04542-0301 (see Fig. 10).

The source RJ is the brightest and most extended of these six detections, with no obvious counterpart in the optical/NIR. Although its extended morphology could be interpreted as an AGN signature, the degeneracy between redshift and dust temperature allows the observed $S_{850 \mu \mathrm{m}} / S_{1.4 \mathrm{GHz}}$ at the position of $\mathrm{RJ}$ to be consistent with a starforming SMG that follows the FIRradio correlation. In any case, the low resolution of the $850 \mu \mathrm{m}$ map makes impossible to determine if $\mathrm{RJ}$ is really contributing to the observed sub-mm emission in this region.

On the other hand, the other five radio detections constitute a strong observational evidence in favor of the merger scenario proposed by Borys et al. (2004). The evidence that supports this conclusion can be summarized as follows:

- if the optical arc and the EROs are multiple images produced by three different regions of a merger at $z=2.9$, the dust obscured material between these regions is expected to be lensed in the same way, but only visible at radio and sub$\mathrm{mm}$. In agreement with this scenario, the shape of the radio emission observed at the sub-mm peak follows the distribution of the optical arc and the ERO images remarkably well (Fig. 7);

- CR1 and CR2 do not have any optical/NIR counterpart. However, they seem to constitute two relatively compact emitting regions embedded in a $\sim 5^{\prime \prime}$ extended source located between E1 and E2. The presence of this extended component (which contributes $38 \%$ of the total radio flux in the sub$\mathrm{mm}$ peak) can only be explained if it is being produced by the dust obscured lensed material in the center of the merger;

- the compact sources E1 and E2 have a high probability of being associated with the images of ERO B, but this probability is considerably lower for E3-B3/C3. A plausible explanation of this result can be provided by the merger scenario, in which the lensed extended component is expected to have a counter image which would be blended with the radio emission associated with E3/C3. This would shift the position of the total observed peak, lowering the probability of identifying E3 and B3/C3 as counterparts;

- the fact that B1, B2 and B3/C3 are not robust $(\geq 95 \%)$ counterparts of E1, E2 and E3, indicates that the radio and NIR emission is being produced at slightly different positions in the sources plane, which can be distinguished thanks to the enhanced resolution provided by the lensing magnification;

- if the $15^{\prime \prime}$ resolution radio map is produced after the CC model of RJ is subtracted from the data, its morphology turns out to be remarkably similar to the sub-mm map (right panel of Fig. 10). This result strongly suggests that the sources E1, E2, CR1, CR2 and E3 are associated with the extended sub$\mathrm{mm}$ emission, allowing us to establish a direct link between SMM J04542-0301 and the merger.

In this paper we have also presented a new lens model of the cluster MS0451.6-0305, produced with the latest version of the LENSTOOL code (Jullo et al. 2007), in which the positions of the ERO images and the arc knots have been used as constrains. The redshifts of the EROs predicted by this model are $z_{\mathrm{B}}=2.93 \pm 0.13$ and $z_{\mathrm{C}}=2.85 \pm 0.06$, in agreement with Borys et al. (2004).

Including the positions of CR1 and CR2 as mirror images in the model significantly degrades the overall rms of the optimization. However, since these two sources are embedded in an extended source (which cannot be included in the lens model due to the current limitations of the LENSTOOL code), it is very difficult to establish a one-to-one identification of structures in the image plane. Therefore, the possibility that CR1 and CR2 are lensed images produced by the dust-obscured core of the merger remains open.

\section{Future prospects}

Radio observations of SMGs have been traditionally used to identify their optical/NIR counterparts, but also to provide estimates of their sizes (Chapman et al. 2004; Biggs \& Ivison 2008). On the other hand, the magnification bias due to the gravitational lensing effect produced by clusters of galaxies has been used to increase the detection rate in sub-mm surveys, and constrain the faint flux end of the submm counts (e.g. Knudsen et al. 2008). The observations presented in this paper, however, illustrate that the prospects for both radio interferometry and strong gravitational lensing in clusters of galaxies to study the internal structure of sub-mm galaxies (SMGs) are also very promising. With the improved sensitivity of e-MERLIN and EVLA, and the revolutionary view of the sub-mm sky that will be provided by ALMA, the enhanced resolution of multiply-imaged submm galaxies might permit us, among other things, to resolve for the first time their different star-forming regions, and help to assess the connection between starburst and AGN processes that seem to coexist in these systems.

SMM04542-0301 constitutes a very promising system to carry out this kind of studies, but there is still one fundamental piece of information that is missing: redshift information for the NIR, radio and submm components. Current millimeter facilities can provide redshift information by detecting (for instance) $\mathrm{CO}$ rotational lines, but the relatively narrow bandwidths of their receivers $(<10 \mathrm{GHz}$ compared with the $115 \mathrm{GHz}$ spacing between $\mathrm{CO}$ rotational lines) makes the search of multiple lines very time consuming, and a prior estimate of the redshift of the source is required. Fortunately, blind $\mathrm{CO}$ redshift observations are now possible thanks to Z-Spec, a broadband $(\sim 115 \mathrm{GHz})$ millimeter/sub-millimeter spectrograph that has been commissioned at the 10.4-m Caltech Submillimeter Observatory (Bradford et al. 2009). Once all the sources that are contributing to the observed sub-mm emission of SMM045420301 have been robustly identified, the increased bandwidth and sensitivity of the new receivers that have been installed on the IRAM $^{11} 30 \mathrm{~m}$ telescope (located on the Pico Veleta, Spain) and the Plateau de Bure Interferometer (located in the French Alps), will be well placed to characterize the physical properties of this system in detail.

Finally note that, since these kind of systems consist of multiple distorted images of the lensed object, a reliable reconstruction of the source is essential for their interpretation. Therefore, to be able to follow this line of research, the technological development in the observational side has to be accompanied by a similar development in the lens modeling techniques in clusters of galaxies, to properly account for the extra complexities associated with extended emission and interferometric observations.

11 Institut de Radioastronomie Millimetrique. 
Acknowledgements. The authors wish to thank Sean Moran and Tadafumi Takata for providing the HST and SUBARU images and source catalogs to compare with the radio observations, and J.P. Kneib for providing the previous LENSTOOL model on MS0451. We are also very grateful to the anonymous referee for his/her useful comments and positive feedback about the paper.

A.B.A. would like to thank Christian Struve and Tom Oosterloo for discussions and helpfull insights regarding the radio data reduction and analysis, Oliver Czoske for helping with the alignment of the different maps and Simona Vegetti for the useful discussions about the lens model (which significantly improved the presentation of the model results). A special mention is reserved for Edo Loenen and Ger de Bruyn, for their crucial contribution in the analysis and interpretation.

This work was supported by the European Community's Sixth Framework Marie Curie Research Training Network Programme, Contract No. MRTN-CT-2004505183 "ANGLES". O.W. was funded by ANGLES and by the Emmy-NoetherProgramme of the Deutsche Forschungemeinschaft, reference Wu 588/1-1. M.L. acknowledges the Centre National d'Études Spatiales (CNES) for their support. The Dark Cosmology Centre is funded by the Danish National Research Foundation.

\section{References}

Alexander, D. M., Bauer, F. E., Brandt, W. N., et al. 2003, AJ, 126, 539 Alexander, D. M., Bauer, F. E., Chapman, S. C., et al. 2005, ApJ, 632, 736 Appleton, P. N., Fadda, D. T., Marleau, F. R., et al. 2004, ApJS, 154, 147

Barger, A. J., Cowie, L. L., Sanders, D. B., et al. 1998, Nature, 394, 248

Barger, A. J., Cowie, L. L., \& Richards, E. A. 2000, AJ, 119, 2092

Beelen, A., Cox, P., Benford, D. J., et al. 2006, ApJ, 642, 694

Berciano Alba, A., Garrett, M. A., Koopmans, L. V. E., \& Wucknitz, O. 2007, A\&A, 462, 903

Biggs, A. D., \& Ivison, R. J. 2008, MNRAS, 385, 893

Blain, A. W., \& Longair, M. S. 1993, MNRAS, 264, 509

Blain, A. W., Smail, I., Ivison, R. J., Kneib, J.-P., \& Frayer, D. T. 2002, Phys. Rep., 369, 111

Borys, C., Chapman, S., Donahue, M., et al. 2004, MNRAS, 352, 759 (B04)

Bradford, C. M., Aguirre, J. E., Aikin, R., et al. 2009, ApJ, 705, 112

Chapman, S. C., Scott, D., Borys, C., \& Fahlman, G. G. 2002, MNRAS, 330, 92

Chapman, S. C., Smail, I., Windhorst, R., Muxlow, T., \& Ivison, R. J. 2004, ApJ, 611,732

Chapman, S. C., Blain, A. W., Smail, I., \& Ivison, R. J. 2005, ApJ, 622, 772

Churchman, L. S., Flyvbjerg, H., \& Spudich, J. A. 2006, Biophys. J., 90, 668

Condon, J. J., Condon, M. A., Gisler, G., \& Puschell, J. J. 1982, ApJ, 252, 102

Cowie, L. L., Barger, A. J., \& Kneib, J.-P. 2002, AJ, 123, 2197

Eales, S., Lilly, S., Gear, W., et al. 1999, ApJ, 515, 518

Elíasdóttir, Á., Limousin, M., Richard, J., et al. 2007, ArXiv e-prints

Franceschini, A., Toffolatti, L., Mazzei, P., Danese, L., \& de Zotti, G. 1991, A\&AS, 89, 285

Garrett, M. A. 2002, A\&A, 384, L19
Garrett, M. A., Knudsen, K. K., \& van der Werf, P. P. 2005, A\&A, 431, L21 Genzel, R., Baker, A. J., Tacconi, L. J., et al. 2003, ApJ, 584, 633 Greve, T. R., Bertoldi, F., Smail, I., et al. 2005, MNRAS, 359, 1165 Hauser, M. G., Arendt, R. G., Kelsall, T., et al. 1998, ApJ, 508, 25 Helou, G., Soifer, B. T., \& Rowan-Robinson, M. 1985, ApJ, 298, L7 Hughes, D. H., Serjeant, S., Dunlop, J., et al. 1998, Nature, 394, 241 Ibar, E., Cirasuolo, M., Ivison, R., et al. 2008, MNRAS, 386, 953 Ivison, R. J., Smail, I., Barger, A. J., et al. 2000, MNRAS, 315, 209 Ivison, R. J., Greve, T. R., Dunlop, J. S., et al. 2007, MNRAS, 380, 199 Jullo, E., Kneib, J.-P., Limousin, M., et al. 2007, New J. Phys., 9, 447 Kettenis, M., van Langevelde, H. J., Reynolds, C., \& Cotton, B. 2006, in Astronomical Data Analysis Software and Systems XV, ed. C. Gabriel, C. Arviset, D. Ponz, \& S. Enrique, ASP Conf. Ser., 351, 497 Kneib, J. P., Mellier, Y., Fort, B., \& Mathez, G. 1993, A\&A, 273, 367

Kneib, J.-P., van der Werf, P. P., Kraiberg Knudsen, K., et al. 2004, MNRAS, 349,1211

Kneib, J.-P., Neri, R., Smail, I., et al. 2005, A\&A, 434, 819

Knudsen, K. K., van der Werf, P. P., \& Kneib, J.-P. 2008, MNRAS, 384, 1611

Knudsen, K. K., Neri, R., Kneib, J.-P., \& van der Werf, P. P. 2009, A\&A, 496, 45

Kodama, T., Tanaka, M., Tamura, T., et al. 2005, PASJ, 57, 309

Kovács, A., Chapman, S. C., Dowell, C. D., et al. 2006, ApJ, 650, 592

Lagache, G., Puget, J.-L., \& Dole, H. 2005, ARA\&A, 43, 727

Lilly, S. J., Eales, S. A., Gear, W. K. P., et al. 1999, ApJ, 518, 641

Limousin, M., Kneib, J.-P., \& Natarajan, P. 2005, MNRAS, 356, 309

Limousin, M., Richard, J., Jullo, E., et al. 2007, ApJ, 668, 643

Lonsdale, C. J., Farrah, D., \& Smith, H. E. 2006, Ultraluminous Infrared Galaxies (Astrophysics Update 2), 285

Meneghetti, M., Bartelmann, M., Jenkins, A., \& Frenk, C. 2007, MNRAS, 381, 171

Michałowski, M. J., Hjorth, J., \& Watson, D. 2009, ArXiv e-prints

Moran, S. M., Ellis, R. S., Treu, T., et al. 2007, ApJ, 671, 1503

Natarajan, P., Kneib, J.-P., Smail, I., \& Ellis, R. S. 1998, ApJ, 499, 600

Natarajan, P., Kneib, J.-P., \& Smail, I. 2002a, ApJ, 580, L11

Natarajan, P., Loeb, A., Kneib, J.-P., \& Smail, I. 2002b, ApJ, 580, L17

Neri, R., Genzel, R., Ivison, R. J., et al. 2003, ApJ, 597, L113

Puget, J.-L., Abergel, A., Bernard, J.-P., et al. 1996, A\&A, 308, L5

Smail, I., Ivison, R. J., \& Blain, A. W. 1997, ApJ, 490, L5

Smail, I., Ivison, R. J., Blain, A. W., \& Kneib, J.-P. 2002, MNRAS, 331, 495

Smail, I., Chapman, S. C., Blain, A. W., \& Ivison, R. J. 2004, ApJ, 616, 71

Smith, G. P., Kneib, J.-P., Smail, I., et al. 2005, MNRAS, 359, 417

Spergel, D. N., Bean, R., Doré, O., et al. 2007, ApJS, 170, 377

Swinbank, A. M., Chapman, S. C., Smail, I., et al. 2006, MNRAS, 371, 465

Swinbank, A. M., Bower, R. G., Smith, G. P., et al. 2007, MNRAS, 376, 479

Tacconi, L. J., Neri, R., Chapman, S. C., et al. 2006, ApJ, 640, 228

Takata, T., Kashikawa, N., Nakanishi, K., et al. 2003, PASJ, 55, 789 (T03)

van der Kruit, P. C. 1971, A\&A, 15, 110

Vlahakis, C., Eales, S., \& Dunne, L. 2007, MNRAS, 379, 1042

Wang, W.-H., Cowie, L. L., van Saders, J., Barger, A. J., \& Williams, J. P. 2007, ApJ, 670, L89

Webb, T. M. A., Lilly, S. J., Clements, D. L., et al. 2003, ApJ, 597, 680 